\title{
ÜBERLEGUNGEN ZUR PROBLEMATIK BEI DER ABGRENZUNG VON SITUATIVERGÄNZUNGEN UND SITUATIVANGABEN
}

\author{
María José Domínguez Vázquez \\ Universidad Santiago de Compostela
}

\begin{abstract}
Abtract:
Ausgehend von der Engelschen Auffassung der Situativergänzungen wird hier an die Adverbialia mittels neu vorgeschlagener analytischer Verfahren herangegangen.

Diese Studie leistet zum einen eine einheitliche Beschreibung bestimmter Verben - stattfinden, gebären, sterben, geschehen, anfangen,öffnen und beginnen - und bietet zum anderen syntaktische, semantische, morphosyntaktische und kommunikative Unterscheidungsmittel für strittige Fälle bei der Unterscheidung zwischen adverbialen Ergänzungen und adverbialen Angaben. Das in diesem Aufsatz Dargestellte kann akzeptiert oder revidiert werden, aber der empirische Teil zeigt eindeutige Ergebnisse.

This article proposes a new analytic approach to adverbials on the basis of Engel's concept of the adverbial component. The study first gives a unified description of certain verbs - stattfinden, gebären, sterben, geschehen, anfangen,öffnen and beginnen - and then proposes syntactic, semantic, morphosyntactic and communicative means for differentiating between adverbial complements and adverbial adjuncts. The description offered in this article can either be accepted or modified, but the empirical part shows very clear results.
\end{abstract}

\section{0 . Vorüberlegungen}

In dieser Studie gehe ich von der Engelschen Auffassung der Situativergänzung ${ }^{1}$ aus und stelle die Frage, ob diese Ergänzung subklassifiziert werden sollte bzw. ob neuere Ergänzungsarten im Rahmen der Adverbialergänzungen angenommen werden sollten. Methodologisch lehnt sich Engel für die Unterscheidung zwischen situativen Ergänzungen und situativen Angaben an das Kriterium der Subklassenspezifik an, und dabei beruft er sich auf die Anapher- und Fragetests, um die Situativergänzungen von anderen Ergänzungen abzugrenzen. Die Beschreibung und Abgrenzung dieser Ergänzung von anderen valenzbezogenen Satzgliedern basiert auf folgenden Kriterien:

Fragetest: Wo?

Anapher: da

Ausdrucksformen:

Präposition + Nominalphrase: Er wohnt in Bremen; Präposition + Pronominalphrase: In ihm befand sich ein Buch; Präpositionaladverb: Darin befand sich ein brauner Briefumschlag; Sonstiges Adverb: (Der Junge wurde in Trier geboren.). - Der Junge wohnt da; Nebensatz: Das Mädchen wohnt, wo es so viel Industrie gibt. ${ }^{2}$

Situativergänzungen sind immer obligatorisch, und deshalb sind alle fakultativen Lokalbestimmungen Angaben. ${ }^{3}$ In Bezug auf die Situativergänzung weist Engel außerdem auf mögliche weitere Unterscheidungen hin: „In den meisten Fällen haben die Situativergänzungen räumliche, seltener auch zeitliche Bedeutung. Bisweilen wird die

${ }^{1}$ Ulrich Engel: Kurze Grammatik der deutschen Sprache, München 2002, S. 25 ff.; Ulrich Engel: Deutsche Grammatik - Neubearbeitung, München 2004, S. $101 \mathrm{ff}$.

${ }^{2}$ Dagegen steht in VALBU (Helmut Schumacher u.a.: VALBU - Valenzwörterbuch deutscher Verben, Tübingen 2004), dass das Verb wohnen keine satzförmige Ergänzung hat.

${ }^{3}$ Vgl. Engel: Kurze Grammatik [Anm. 1], S. 26. 
Ansicht vertreten, daß es auch kausale (und eventuell noch weitere) Situativergänzungen gebe. Diese Frage soll hier offengelassen werden. “ 4

Den Zitaten von Engel und Engel/Savin in „Valenzlexikon deutsch-rumänisch“5 kann entnommen werden, dass die Situativergänzung möglicherweise weiter differenziert werden könnte. ${ }^{6}$ Ein Beispiel dafür ist die Aufnahme einer neuen Ergänzung, nämlich der Modifikativergänzung, in das Ergänzungssystem von Engel $^{7}$, die auf der spezifischen Anapher auf diese Art basiert. ${ }^{8}$. Von dieser bei Engel erkennbaren Überlegung und Erweiterung seiner Ergänzungstypologie geht meine Untersuchung aus. Ich möchte hierbei prüfen, ob eine Klassifikation mit weiteren Ergänzungen wie Lokal- ${ }^{9}$, Temporal- und Kausalergänzung zu begründen ist und zusätzlich bei welchen Verben bzw. unter welchen Umständen sie vorkommen. Dazu werde ich in dieser Arbeit Beschreibungsparameter vorschlagen.

Meine Untersuchung ist folgendermaßen gegliedert. An erster Stelle wird die Analyse der temporalen, kausalen und weiterer Satzglieder in unterschiedlichen Valenzwerken dargestellt und verglichen. Unterscheidungskriterien werden eingeführt, die zur Untersuchung der vorliegenden Thematik dienen. Die Darlegung dieser Kriterien hat nur als erste Annäherung an den Untersuchungsgegenstand zu gelten, denn in dieser Arbeitsphase wird keine Stellung zur Adäquatheit dieser Unterscheidungsmittel genommen. An zweiter Stelle werden die in Valenzwerken enthaltenen Verben, bei denen eine Situativergänzung vorliegt, einzeln analysiert. Anhand der gesamten Ergebnisse wird schließlich zwei Schwerpunkten nachgegangen: einerseits inwiefern die vorgestellten Unterscheidungsmittel bzw. -kriterien funktionsfähig sind (tragen sie zur Unterscheidung der situativen Ergänzungen von den situativen Angaben bei?) und andererseits, ob eine Lokal-,Temporal- oder Kausalergänzung $\mathrm{zu}$ bestimmen ist und bei welchen Verben diese Ergänzungen vorkommen.

\section{Betrachtung der Situativergänzung in Valenzlexika und Monographien zur Valenz}

Wenn man Grammatiken, linguistische Monographien und Valenzwörterbücher aufmerksam liest, wird man finden, dass einige von ihnen Lokal-, Temporal- und Kausalergänzungen ansetzen.

Engel/Savin im „Valenzlexikon deutsch-rumänisch“ ordnen den folgenden Verben unterschiedliche Arten von Situativergänzungen zu: wohnen (lokal), abgeben (lokal), arbeiten (lokal), sich ereignen (lokal, temporal, kausal), regieren (lokal, temporal), sterben (lokal,

\footnotetext{
${ }^{4}$ Ulrich Engel: Syntax der deutschen Gegenwartssprache, 1/3, Berlin 1977/1994, S. 161.

${ }^{5}$ Ulrich Engel, Emilia Savin: Valenzlexikon deutsch-rumänisch, Heidelberg 1983.

${ }^{6}$ Vgl. auch Engel: Kurze Grammatik [Anm. 1], S. 26.

${ }^{7}$ Ulrich Engel: Deutsche Grammatik [Anm. 1], S.103.

${ }^{8}$ Vgl. auch die Betrachtung der Modalergänzung in María José Domínguez Vázquez: Die Präpositivergänzung im Deutschen und im Spanischen. Zur Bedeutung der Präpositionen, Frankfurt 2005.

${ }^{9}$ In Bezug auf die Lokalergänzung oder Situativergänzung soll hier geprüft werden, mit welchen Verben sie auftritt.
} 
temporal), stattfinden (lokal, temporal). Bei anfangen und geschehen gilt das temporale, das lokale und das kausale Glied als Angabe. Das Verb sich benehmen wird hier nicht behandelt.

In „Verbvalenz und Satzbaupläne“, einer kontrastiven Studie Deutsch-Rumänisch, erläutert Speranta Stănescu in Bezug auf die Situativergänzung, dass ,alle Elemente dieser Klasse ein Geschehen ,situieren', es also in eine Situation einbetten. Wenn man indessen unter Situierung ganz generell die Eingliederung eines Geschehens in eine Kosituation versteht, lassen sich mit Hilfe der Anaphern mehrere Untergruppen ermitteln. “10 Folgende Ergänzungen bilden dannn Subklassen innerhalb der Situativergänzung:

Lokalergänzung: Hans wohnt in Bukarest/in einem neuen Haus/dort/hier. Dazu zählt Stănescu auch die Verben verstecken, parken, nehmen, verteilen, vorlesen, bedanken, beschweren, bewerben, entkommen, gehen, hängen, geben <es $>$, ankommen $<e s\rangle$, wohnen. Temporalergänzung: Das Fest beginnt, wann du willst/um 10 Uhr/genau dann/jetzt. Dazu auch die Verben angehen, beginnen, sich ereignen. Modalergänzung: Er benimmt sich, wie es sich gehört/ganz unmöglich/entgegen allen Erwartungen/so. Andere Verben, die eine Modalergänzung im Satzbauplau enthalten, sind weiter: lohnen, anfangen, beginnen, kommen, nützen, stehen, verlaufen, gefallen $<e s>$, gehen $<e s>$. Kausalergänzung: Der Mord geschah, weil er eifersüchtig war/aus Eifersucht/deshalb. Das Verb geschehen benötigt nach Stănescu eine Kausalergänzung. ${ }^{11}$

In „Verben in Feldern“12, einem Valenzwörterbuch zur Syntax und Semantik deutscher Verben, kommt bei geschehen keine andere Ergänzung als die Subjektergänzung in Betracht. Bei beginnen, enden, anfangen und stattfinden wird bei der Verbvalenz eine fakultative Adverbialergänzung angesetzt.

In „Braucht die Valenzgrammatik Objekte und Adverbiale?“ unterscheidet Tarvainen ${ }^{13}$ innerhalb der Situativergänzung drei Ergänzungen: Zeitergänzung- Die Sitzung dauerte eine Stunde. $^{14}$ / Begründungsergänzung- Der Unfall geschah aus Unachtsamkeit. / Raumergänzung- Ich wohne in Jyväskylä.

Für die deutsche und die polnische Sprache möchte ich mich auf das „Wörterbuch zur Valenz deutscher und polnischer Verben" ${ }^{15}$ beziehen. Bei anfangen und beginnen wird eine Modalergänzung, aber keine Temporal- oder Lokalergänzung anerkannt. Bei sterben erfüllt das temporale Satzglied die Funktion einer Angabe. Bei sich ereignen erfüllt das temporale, lokale und kausale Satzglied die Funktion einer Angabe. Bei stattfinden handelt es sich bei

\footnotetext{
${ }^{10}$ Speranta Stănescu: Verbvalenz und Satzbaupläne, Heidelberg 1986, S. 84 f.

${ }^{11} \mathrm{Zu}$ dieser Gruppe der Situativergänzung zählt Stănescu auch die Komparativergänzung und die Maß- oder Erstreckungsergänzung, auf die ich hier nicht eingehen werde.

${ }^{12}$ Helmut Schumacher u.a.: Verben in Feldern. Valenzwörterbuch zur Syntax und Semantik deutscher Verben, Berlin, New York 1986.

${ }^{13}$ Kalevi Tarvainen: Braucht die Valenzgrammatik Objekte und Adverbiale?, in: Valenzen im Kontrast: Ulrich Engel zum 60. Geb., hg. v. Pavica Mrazovic, Wolfgang Teubert, Heidelberg 1988, S. 380.

${ }^{14}$ Tarvainens Zeitergänzung entspricht in diesem Fall der Expansivergänzung von Engel (vgl. Engel: Kurze Grammatik [Anm. 1] und die Raumergänzung der Situativergänzung (lokal) von Engel. Eine Entsprechung der Begründungsergänzung von Tarvanien kommt bei Engel nicht vor.

${ }^{15}$ Leslaw Cirko, Norbert Morciniec, Ryszard Ziobro: Wörterbuch zur Valenz deutscher und polnischer Verben, Wroclaw 1995.
} 
den temporalen und lokalen Satzgliedern um eine Angabe. Bei geschehen erfüllen temporale, kausale und lokale Satzglieder die Funktion einer fakultativen Ergänzung. Bei öffnen gilt das temporale Satzglied als eine Temporalergänzung. Das Verb sich benehmen ist in diesem Wörterbuch nicht verzeichnet.

Maria Teresa Bianco arbeitet im „Valenzlexikon Deutsch-Italienisch“16 mit einer Temporalund einer Lokalergänzung und zieht kausale und modale Ergänzungen nicht in Betracht. In Bezug auf diese Ergänzungen und die nach Engel definierte Situativergänzung führt sie den folgenden Unterschied an: a) Die Situativergänzung von Engel, insofern sie eine Tatsache, einen Umstand oder ein Ereignis zu einem bestimmten Zeitpunkt angibt, wird als Temporalergänzung klassifiziert; b) Die Situativergänzung von Engel, insofern sie eine Tatsache, einen Umstand oder ein Ereignis an einem bestimmten Ort angibt, wird als Lokalergänzung verstanden. In Biancos Lokalergänzung wird Engels Direktivergänzung miteinbezogen.

In „VALBU. Valenzwörterbuch deutscher Verben“ umfasst der Terminus Adverbativergänzung unterschiedliche Ergänzungen, darunter die Subklasse des Räumlichen und Zeitlichen, die Subklasse des Punktuellen und Durativen und die Subklassen „Mittel“, „Art und Weise“, „Menge“, „Zweck“ und „Zusammenhang“. ${ }^{17}$ Beim Verb beginnen tritt das Satzmuster Subjektergänzung Adverbativergänzung auf, wobei die Adverbativergänzung semantisch als irgendwo, irgendwann oder irgendwie beschrieben wird. Bei beginnen liegen die semantischen Begleiter Ort und Zeit vor, genauso wie bei stattfinden. Geschehen wird in einer der Realisierungen mit der Bedeutung irgendwie wiedergegeben, und außerdem führt eine der Definitionen dieses Verbs auf veranstalten zurück. Bei gebären und sterben werden ebenfalls Adverbialergänzungen beschrieben, genauso wie bei öffnen.

Einerseits kann man an den bereits angeführten Beschreibungen erkennen, dass die Sprachwissenschaftler diese Satzglieder unterschiedlich kategorisieren oder als Übergangselemente zwischen Ergänzung und Angabe verstehen. ${ }^{18}$ Andererseits schreiben einige Wissenschaftler dem, was andere als fakultative Ergänzung verstehen, den Status einer Angabe zu. So sagt Engel:

Die Situativergänzung gehört zu den wenigen Ergänzungen, die neben gleichlautenden situativen Angaben stehen. Das schafft Abgrenzungsprobleme vor allem deshalb, weil lokale und temporale Bestimmungen sozusagen von Haus aus, angabeverdächtig sind, sie lassen sich schließlich mit beliebigen Verben kombinieren. Deshalb rechnen wir von den situativen Elementen (...) nur die obligatorischen zu den Ergänzungen; alle fakultativen Situativelemente sind demnach Angaben.

Unbestreitbar ist freilich, daß bestimmte Verben - so arbeiten, leben, spielen und nicht wenige andere - eine Art von Affinität zu Situativelementen aufweisen, so daß viele Grammatiker auch fakultative Situativergänzungen zulassen: Sicher käme ein skalarer Übergang zwischen Ergänzungen und Angaben gerade im Bereich der Situativelemente der sprachlichen Intuition eher entgegen als eine brüske Dichotomisierung. ${ }^{19}$

\footnotetext{
${ }^{16}$ Maria Teresa Bianco: Valenzlexikon Deutsch-Italienisch, Dizionario della valenza verbale, 2 Bde., Heidelberg 1996.

${ }^{17}$ Schumacher u.a. [Anm.2].

${ }^{18}$ Ebd, S.34.

${ }^{19}$ Engel [Anm. 4], S. 161.
} 
Dazu bleibt immer noch die Frage offen, bei welchen Verben tatsächlich diese Satzglieder als Ergänzung oder als Angabe gelten. Dass selbstverständlich alle Ereignisse zu einem bestimmten Zeitpunkt, an einem bestimmten Ort, auf eine bestimmte Weise, aus irgendeinem bestimmten Grund usw. geschehen, sollte uns nicht darüber hinwegtäuschen, dass die hier zu analysierenden ,Bestimmungen nicht immer die Funktion einer Angabe erfüllen, wie Beispiele der Art Renate wohnt in Mannheim belegen.

Da sich die Wissenschaftler darüber nicht einig sind, werde ich einige der bereits erwähnten Verben analysieren, was mir auch ermöglichen wird, auf die Frage nach dem Ergänzungsbzw. Angabestatus bestimmter Satzglieder einzugehen. Für das Recherchieren dieser Verben habe ich eine Beispielsammlung von Texten der geschriebenen deutschen Sprache zusammengestellt, die ich aus dem Programm Cosmas des Instituts für deutsche Sprache, Mannheim, gewonnen habe. Die Belege stammen aus den Jahren ab 1989.

\section{Kriterien zur Abgrenzung von adverbialen Angaben und adverbialen Ergänzungen}

\subsection{Syntaktische und semantische Kriterien}

\subsubsection{Die Spezifizität}

Die Spezifizität oder Aspezifizität von Satzgliedern, die von Engel seit 1970 als Unterscheidungskriterium für Angabe und Ergänzung verwendet wird, gilt hier als syntaktisches Kriterium. In Anlehnung an Engel ${ }^{20}$ möchte ich aber mein Vorgehen auf folgende Überlegungen stützen:

Bei einigen Verben sind eine Akkusativ-, Dativ- oder Präpositivergänzung valenzbezogen. Dies erklärt u.a. Andresen ${ }^{21}$ mittels ihrer Kommutationsprobe. Bei ihrem Versuch, Angaben und Ergänzungen zu unterscheiden, ergibt sich, dass schon ein Wechsel des Verbs bedeutende Auswirkungen hat ${ }^{22}:{ }^{*} E r$ wohnt das ganze Jahr - Er wohnt das ganze Jahr in Mannheim. Im Vergleich zu Er bewohnt das Haus. Dazu gelten bestimmte Sätze ohne die entsprechende Ergänzung als agrammatisch, z.B. *Er mag jetzt - Er mag es jetzt; * Er geht im Kino - Er geht ins Kino.

Bei anderen Verben werden jedoch die bereits genannten Ergänzungen (Akkusativ-, Dativund Präpositivergänzung) nicht zugelassen, stattdessen allerdings andere ,Bestimmungen . Hätten wir die Beispiele Ein Unfall ist geschehen und Der Unfall ist gestern geschehen, könnte man behaupten, dass im zweiten Fall eine Ergänzung, im Konkreten eine Temporalergänzung, vorkommt. Warum? Meine Behauptung stützt sich auf den Fragetest: a)

\footnotetext{
${ }^{20}$ Ulrich Engel: Deutsche Grammatik, Heidelberg 1988.

${ }^{21}$ Helga Andresen: Ein methodischer Vorschlag zur Unterscheidung von Ergänzung und Angabe im Rahmen der Valenztheorie, in: Deutsche Sprache, H. 1, 1973, 49-63.

${ }^{22}$ Diese Methode bezieht sich nur auf die Kasusergänzungen, wobei Probleme bei der Einstufung von Adverbialia bestehen. Mit dieser Methode kann man nur zu dem Ergebnis kommen, dass ein Austausch des Verbs keine morphologische Konsequenz für die Angaben verursacht.
} 
Ein Unfall ist geschehen: Was ist geschehen? Ein Unfall. b) Der Unfall ist gestern geschehen: Wann ist der Unfall geschehen? Gestern.

Außerdem verursacht das Weglassen von „gestern“ bei b. eine andere Bedeutung als die von Der Unfall ist gestern geschehen, d.h. es gibt unterschiedliche Verbrealisierung (vgl. dazu 2.1.2. und 2.2.). Dies soll im Weiteren vertieft werden.

\subsubsection{Eliminierungstest und Reduktionstest}

Lange wurden die Angaben und Ergänzungen mittels der Abstrichmethode ${ }^{23}$ unterschieden. Diese Methode beruht auf der Annahme, dass weglassbare Elemente, nach deren Elimination der verbleibende Satz grammatisch ist, als Angaben aufzufassen sind. Seit 1969 vertreten Gerhard Helbig und Wolfgang Schenkel ${ }^{24}$ den Eliminierungstest und Gisela Zifonun und andere $^{25}$ den Reduktionstest.

Im Folgenden werde ich mich mit dem Reduktionstest befassen. Der Reduktionstest prüft die Fixiertheit, die den Kriterien der Weglassbarkeit bzw. Nicht-Weglassbarkeit entspricht. Eine Ergänzung ist unter diesem Kriterium nicht weglassbar, d.h. ++ fixiert, oder nur weglassbar, d.h. + fixiert, wenn sie kontextuell oder semantisch markiert ist. Eine Ergänzung ist + fixiert bei Phänomenen wie Vorerwähntheit, situativer und lexikalisierter Ellipse, Kontrastbetonung, Modalisierung, Ausdruck von Generizität oder Habitualität, z.B.: Behandelt Sie Dr. Fritz? ${ }^{*} J a$, er behandelt; Verzichtest du auf die Teilnahme? - Ja, ich verzichte. (Beispiel für Weglassbarkeit bei Vorerwähntheit $\left.{ }^{26}\right)$. Bei diesem Test geht man von einer vollständigen kommunikativen Minimaleinheit (Vollsatz) aus. Es geht darum, die kommunikative Minimaleinheit jeweils um eine Phrase zu reduzieren: „Kann das Resultat der Reduktion nicht mehr als Ausdruck einer Proposition gelten oder verändert sich bei der Reduktion die Interpretation des verbalen Prädikatausdrucks, so ist die Phrase, um die reduziert wurde, ein Komplement, sofern sie nicht Teil des Verbalkomplexes ist.“27

Die Frage lässt sich wie folgt kurz zusammenfassen: Ist der verbleibende Ausdruck ohne die Phrase unvollständig? Wenn die Antwort ja lautet, dann handelt es sich um eine Ergänzung. Bei einigen Reduktionen ergeben sich keine Schwierigkeiten, denn diese Reduktion führt zu ungrammatischen Ausdrücken. Falls die Bedeutung des resultierenden Ausdrucks anders zu interpretieren ist, handelt es sich ebenfalls um eine Ergänzung. Das ist am folgenden Beispiel zu veranschaulichen: a. Hans, der schwere Junge, sitzt gerade in der Badewanne. b. Hans, der schwere Junge, sitzt gerade. In der Badewanne gilt als Ergänzung, denn mit dem Weglassen dieses Syntagmas geht eine andere Bedeutung bzw. ein anderer Gebrauch einher. Beim Fall b. wird hier sitzt im Sinne von ist Strafgefangener verwendet.

Der Reduktionstest soll bei den hier zu analysierenden Verben angewandt werden (vgl. 3.).

\footnotetext{
${ }^{23}$ Vgl. Leo Weisgerber: Vom Weltbild der deutschen Sprache, Düsseldorf 1954; Duden. Grammatik der deutschen Gegenwartssprache, 3 Bde., hg. v. Paul Grebe, Mannheim 1959/1973.

24 Gerhard Helbig, Wolfgang Schenkel: Wörterbuch zur Valenz und Distribution deutscher Verben, Leipzig/Tübingen ${ }^{7} 1983$.

${ }^{25}$ Gisela Zifonun u.a.: Grammatik der deutschen Sprache, 3 Bde., Berlin, New York 1997.

${ }^{26}$ Ebd., S. 1031.

${ }^{27}$ Ebd., S. 1043.
} 


\subsection{Die Valenzrealisierungen des Verbs}

Für die Bestimmung unterschiedlicher Valenzrealisierungen eines Verbs sollte meines Erachtens zunächst eine erste Grenze zwischen intransitiven Verben ${ }^{28}$ (Das Kind ist gestern gestorben) und transitiven Verben gezogen werden. Bei den transitiven Verben kann man zwischen transitiven und intransitiven Valenzrealisierungen (Ich habe ein Kind geboren/Das Kind ist gestern geboren) unterscheiden. Es soll hier der Frage nachgegangen werden, wie häufig lokale, temporale, kausale und modale Satzglieder bei den intransitiven Verben bzw. intransitiven Realisierungen vorkommen, oder umgekehrt welche und warum genau diese Satzglieder weggelassen werden. Bei den transitiven Verben ist $\mathrm{zu}$ prüfen, bei welcher Realisierung die lokalen, temporalen, kausalen und modalen Satzglieder erwartet werden und wie regelmäßig diese Satzglieder vorkommen. ${ }^{29}$

Die möglichen unterschiedlichen Valenzrealisierungen eines Verbs sind nach folgenden Unterscheidungskriterien zu erkennen: der Fragetest, der Reduktionstest und die semantischen Eigenschaften, die dem Subjekt zugesprochen werden können. ${ }^{30}$ Die Anwendung dieser Kriterien soll am Beispiel vom Verb beginnen veranschaulicht werden:

A. ,etwas beginnt tatsächlich/jetzt ${ }^{e}$. Intransitive Valenzrealisierung. Bsp: Einige Veranstaltungen beginnen. Subjektergänzung: Nicht-Agens-Rolle ([akt]/[stat $])^{31}$, -bestimmt, bekannt. Die Bedeutung ist ,etwas geschieht ${ }^{\circ}$ und in diesem Fall kann man mit Was beginnt? nachfragen. Es handelt sich hier um eine einwertige Valenzrealisierung, da hier nur eine Subjektergänzung vorliegt.

B. ,etwas beginnt in einer bestimmten Situierung, etwas fängt an `. Intransitive Valenzrealisierung. Bsp: Die Veranstaltungen beginnen um 8 Uhr. Subjektergänzung: NichtAgens-Rolle ([akt]/[stat]), +bestimmt, +bekannt. Die Bedeutung lautet ,etwas beginnt zu einem Zeitpunkt ${ }^{\circ}$. In diesem Fall kann man mit Wann beginnen die Veranstaltungen? nachfragen. Es handelt sich hier um eine zweiwertige Valenzrealisierung.

\footnotetext{
${ }^{28}$ Ich verwende den Begriff transitiv/intransitiv in einem weiten Sinn, d.h. unter Transitivität verstehe ich nicht nur die passivfähigen Akkusativverben, sondern auch die Dativverben, Genitivverben und Präpositionalverben. Näheres über Transitivität in diesem Sinne bei Engel (Ulrich Engel: Deutsche Gramatik, Heidelberg ${ }^{3} 1996$, S. 391).

${ }^{29}$ In Bezug auf die unterschiedlichen Valenzrealisierungen sollen auch semantische Rollen und ihre semantischen Restriktionen mitberücksichtigt werden. Meines Erachtens kann man auf diese Weise die Verbrollen bestimmen, und dadurch kann man das untersuchte Verb mit anderen der gleichen Klasse vergleichen, um die subklassenspezifischen Rollen zu bestimmen (vgl. dazu 2.4.).

${ }^{30}$ Darunter verstehe ich die relationale und kategorielle Bedeutung. Die kategorielle Bedeutung wird nach Engel ([Anm. 5], S. 865) wie folgt definiert: sie ist der „,kategorielle Teil der kombinatorischen Bedeutung, legt (zum Teil) die inhärente Bedeutung umgebender Elemente fest, ist wesentlicher Bestandteil der Inhaltsvalenz eines Wortes“. Die relationale Bedeutung wird nach Engel wie folgt beschrieben: sie ist der ,relationale Teil der kombinatorischen Bedeutung, legt die semantische Relation zwischen einem Wort und seiner Umgebung fest, ist wesentlicher Bestandteil der Inhaltsvalenz dieses Wortes“ (ebd.). Beide Bedeutungen, die relationale und die kategorielle, gehören zur kombinatorischen Bedeutung, die nach Engel die Bedeutung ist, „die für die Umgebung eines Wortes gilt (Gegensatz: inhärente Bedeutung); soviel wie Inhaltsvalenz des betreffenden Wortes“ (ebd.).

${ }^{31}$ Als semantische Restriktion gilt hier ein Sachverhalt, der entweder eine Eigenschaft, ein Zustand ([stat]) oder ein Vorgang, ein Geschehensablauf, eine Tätigkeit ([akt]) ist (ebd., S. 358 f.).
} 
C. ,jemand beginnt etwas ${ }^{e}$, jemand beginnt mit etwas'. Transitive und Präpositionale Valenzrealisierung. Bsp: Ich beginne mit einer neuen Arbeit. Subjektergänzung: Agens-Rolle $([\text { hum }] /[\text { inst }])^{32},+/-$ bestimmt, +/- bekannt. Als Bedeutung gelten ,Ich beginne etwas , ,Ich beginne mit etwas ${ }^{e}$. In diesem Fall kann man Womit beginnst du? nachfragen.

Ich möchte bei der Valenzrealisierung B. den Reduktionstest durchführen, wobei sich der Ausdruck Die Veranstaltungen beginnen ergibt. Dieser Ausdruck kann als grammatisch nur in dem Fall einer Umschreibung, etwas geschieht tatsächlich/jetzt ${ }^{\circ}$ verstanden werden. In diesem Fall können nur Ja/Nein-Fragen formuliert werden, der Fragetest lautet: Beginnen die Veranstaltungen? Meiner Ansicht nach gibt dieser resultierende Satz eine andere Bedeutung als der Ausgangssatz - Die Veranstaltungen beginnen um $8 \mathrm{Uhr}$ - wieder.

Würde man den Reduktionstest bei anderen Beispielen wie a. Ich las das Buch gestern Abend. - b. Ich las das Buch durchführen, kämen wir dagegen zum Ergebnis, dass der resultierende Ausdruck b. genauso wie der Ausdruck a. zu interpretieren ist. Der einzige Unterschied liegt darin, dass sich ein Informationsverlust ergibt, aber keine andere kommunikative Einheit, d.h. es handelt sich um die gleiche Valenzrealisierung ${ }^{33}$ (vgl. auch 3.5.C). Dies halte ich für ein grundlegendes Faktum, das die Auffassung unterstützt, dass mehrere Bedeutungsvarianten eines Verbs vorliegen, wenn das gleiche Verb der Zahl oder Art nach verschiedene Mitspieler hat. ${ }^{34}$ Dazu vertritt Eva Breindl die Meinung, „,dass man lieber von polysemen Verben oder Bedeutungsvarianten eines Verbs mit unterschiedlicher Valenzstruktur sprechen sollte““. ${ }^{35}$

Dies soll bei jedem der hier präsentierten Verben analysiert werden.

\subsection{Morphosyntaktisches Kriterium. Die Topologie}

Die Unterscheidung zwischen intransitiven und transitiven Valenzrealisierungen ermöglicht es uns zu analysieren, ob davon abhängig unterschiedliche Satzgliedpositionen bei den temporalen, lokalen, modalen und kausalen Satzgliedern festzustellen sind. Hierbei beabsichtige ich keine vollständige Analyse, sondern einen Vergleich zwischen intransitiven und transitiven Verbrealisierungen. Wenn temporale, lokale, modale und kausale Satzglieder bei intransitiven oder transitiven Verbrealisierungen andere Positionen im Satz besetzen, sollte man sich überlegen, ob die hier untersuchten Satzglieder zu den Ergänzungen oder zu den Angaben gehören. ${ }^{36}$ Die Analyse soll hierbei die Besetzung des Vorfelds oder des Mittelfelds zum Gegenstand haben und dazu berufe ich mich auf Engel ${ }^{37}$ und Zifonun u.a. ${ }^{38}$

\footnotetext{
32 Es handelt sich um materielle semantische Restriktionen, die entweder [hum] (,menschlich ${ }^{c}$ ) oder [inst] (,Institutionen ${ }^{e}$ ) sind (ebd.).

${ }^{33} \mathrm{Zu}$ bemerken ist noch, dass bei diesen Verben temporale, lokale oder kausale Satzglieder vorkommen. In diesem Zusammenhang lehne ich mich an Renate Steinitz: Adverbial-Syntax, Berlin 1969, S. 13 ff., 26 ff. an, die sagt, dass bei einigen Verben die Verbvalenz nur das Vorkommen des Gliedes und nicht seine Art bestimmt.

${ }^{34}$ Vgl. Helbig, Schenkel [Anm. 24], S. 50.

${ }^{35}$ Eva Breindl: Präpositionalobjekte und Präpositionalobjektsätze im Deutschen, Tübingen 1989, S. 18.

36 Aus der gesamten Beispielsammlung habe ich mich für die Satzstellungsuntersuchung auf Hauptsätze (Aussagesätze) beschränkt.

${ }^{37}$ Engel [Anm. 20], S. 322 ff.

${ }^{38}$ Zifonun [Anm. 25], S. 1507 ff.
} 
Eine Vertiefung der topologischen Prinzipien wird von mir nicht beabsichtigt. Es genügt festzustellen, dass Angaben und Ergänzungen nicht beliebig im Satz positioniert werden können und dass bestimmte Beschränkungen für die Besetzung des Vorfelds bestehen. ${ }^{39}$ Meiner Ansicht nach kann dieses Kriterium nur zuverlässige Ergebnisse gewährleisten, wenn eine repräsentative Beispielsammlung zur Analyse herangezogen wird.

\section{4 . Das kommunikative Kriterium}

Nach Charles J. Fillmore ${ }^{40}$, Hans Jürgen Heringer ${ }^{41}$ und Angelika Storrer ${ }^{42}$ sind die Ergänzungen als subklassenspezifische Verbrollen und +/-perspektivierungsfixiert zu verstehen. Die perspektivierungsfixierten Rollen können als Teil sprachlicher Äußerungen im Rahmen einer bestimmten Szene und anhand von Korpussätzen festgestellt werden. Storrer spricht sich dafür aus, dass es perspektivierungsfixierte verbspezifizische Rollen in einer Situation gibt, ,wenn mit der Wahl des betreffenden Verbs diese Rolle auf jeden Fall zu realisieren ist und somit die ihr entsprechende Situationsrolle auf jeden Fall perspektiviert wird“. ${ }^{43}$ Christa Dürscheids Ansatz ${ }^{44}$ knüpft daran an: „Außerhalb der Perspektive sind alle die ontologischen Entitäten, die zwar am Sachverhalt beteiligt sind, aber in der sprachlichen Präsentation des Sachverhalts ausgeblendet, d.h. im Prozess der Perspektivierung nicht versprachlicht werden. “45

Der innersprachliche Perspektivierungsprozess regelt die relative Anordnung der Rollen untereinander und baut eine Perspektive auf. Diese Rollen können sprachlich in unterschiedlicher Weise perspektiviert sein. Wie sie innersprachlich perspektiviert werden, erläutert Elisabeth Leiss ${ }^{46}$ :

\footnotetext{
${ }^{39}$ Vgl. Christa Dürscheid: Syntax. Grundlagen und Theorien, Wiesbaden 2000. Diese Restriktionen sind: a) „nicht nominativische Pronomina können nur dann im Vorfeld stehen, wenn sie betont sind. [...] Das gilt auch für unbetonte Partikeln wie halt, aber und ja und für den freien Dativus Ethicus“ (ebd., S. 97: *Es habe ich auf den Stuhl gelegt); b) es ist auch möglich, „dass nur ein infinites Verb oder ein Verbzusatz das Vorfeld besetzt“ (ebd., S. 98: Vergessen werde ich das nie).

${ }^{40}$ Charles .J. Fillmore: The Case for Case Reopened, in: Syntax and Semantics, hg. v. Peter Cole, Jerrold M. Sadock, New York, San Francisco, London 1977 („Grammatical Relations“, S. 59-81); Charles J. Fillmore: Scenesand-Frames Semantics, in: Linguistic Structures Processing, hg. v. Antonio Zampolli, Amsterdam, New York, Oxford 1977, S. 55-81.

${ }^{41}$ Hans Jürgen Heringer: Neues von der Verbszene, in: Pragmatik in der Grammatik, hg. v. Gerdhard Stickel, Düsseldorf 1984, S. 34-64.

42 Angelika Storrer: Verbvalenz. Theoretische und methodische Grundlagen ihrer Beschreibung in Grammatikographie und Lexikographie, Tübingen 1992.

${ }^{43}$ Ebd., S. 285.

${ }^{44}$ Christa Dürscheid: Zur Vorfeldbesetzung in deutschen Verbzweit-Strukturen, Trier 1989, S. 248.

${ }^{45} \mathrm{Um}$ das bereits genannte Vorgehen zu erhellen, möchte ich beispielsweise auf das Verb verkaufen eingehen. Bei diesem Verb liegt eine bestimmte Szene vor: der Verkäufer, der Kunde, der Preis usw., wobei temporale, kausale, modale Hinweise hinzu kommen können. Wenn man dagegen z.B. eine Todesanzeige untersuchte, käme man zum Ergebnis, dass die lokale und temporale Information am wichtigsten ist.

46 Elisabeth Leiss: Die Verbalvalenz des Deutschen. Ein Beitrag zur Theorie der sprachlichen Kategorisierung, Berlin, New York 1992, S. 102.
} 
Nehmen wir als Beispiel folgende Szene oder folgende beide ,Verbszenen ${ }^{`}$ und stellen uns vor, wir sollten diese Szene in eine filmische Szene umsetzen:

(1) Hausmeister Soundso ruft die Feuerwehr an.

(2) Die Feuerwehr wird von Hausmeister Soundso angerufen.

Es ist in diesem Fall nur möglich, einen der beiden Standpunkte als Zentrum und als Drehort zu wählen. (1) gibt dabei eine andere Drehanweisung als (2).

Einige Einheiten werden häufiger ausgedrückt und am häufigsten von den Hörern erwartet, und daher stellt sich mir die Frage, wie es mit den Ereignisverben steht, d.h. was wird erwartet, wenn Verben wie stattfinden, geschehen u.a. verwendet werden. Daran anschließend möchte ich auf die Anwendung des Erwartbarkeits- und Vorkommenshäufigkeitsprinzips eingehen.

Um die temporalen, lokalen und kausalen Satzglieder als Ergänzung oder als Angabe zu klassifizieren, könnte man vom Prinzip der Erwartbarkeit ${ }^{47}$ und der Vorkommenshäufigkeit eines Satzgliedes im Satz ausgehen. Dieses Vorgehen ist bereits auf Ulrich Engel/Helmut Schumacher ${ }^{48}$ zurückzuführen, die z.B. bei ankommen das Auftreten einer Ergänzung durch ihre hohe Erwartbarkeit begründen. Um den Status von Satzgliedern als Angabe oder als Ergänzung zu kategorisieren, sagt Engel außerdem in „Die deutschen Satzbaupläne“449 Folgendes: „Ist bei Vorhandensein dieses Verbs ,handeln' mit einer Artbestimmung zu rechnen, ist eine solche Artbestimmung vorhersagbar?“50. Weiter heißt es: „Wird die Frage mit ,ja ${ }^{e}$ beantwortet, so liegt eine Ergänzung vor, andernfalls eine Angabe. “51 Diese Erwartbarkeit kann als Vorkommenswahrscheinlichkeit zusammengefasst werden: „Obligatorik ist ja nichts anderes als eine extreme Ausprägung der Wahrscheinlichkeit, eben eine Wahrscheinlichkeit von 100\%. Mit der Erwartbarkeit bzw. Vorkommenswahrscheinlichkeit ist nun allerdings ein Kriterium eingeführt, das der Diskussion um die Abgrenzbarkeit von E und A eine neue Wendung gibt.“ 52

Die Vorkommenshäufigkeit wird der Belegsammlung entnommen. Nach diesem Kriterium kann man prüfen, welche Verbrollen und in welcher Anzahl diese in einer bestimmten Szene vorkommen können, man kann jedoch nicht feststellen, inwiefern diese Rollen subklassenspezifisch sind. Um die Subklassenspezifität bestimmen zu können, sollte man meines Erachtens nach Arten von Verben vorgehen, z.B.: Verben des Sich-Befindens, Ereignisverben usw. Falls die Anzahl bei einer bestimmten Gruppe hoch ist und falls weitere Bedingungen ${ }^{53}$ erfüllt werden, könnte man zu der Feststellung kommen, es handelt sich um subklassenspezifische Verbrollen, die ihrerserseits perspektivierungsfixiert sein können oder nicht.

Nach den oben genannten Vorüberlegungen gehe ich folgenden Fragen nach:

Kann man von einer Temporal-, Kausal-, Lokal- und Modalergänzung sprechen?

\footnotetext{
${ }^{47}$ Vgl. Schumacher [Anm. 12].

${ }^{48}$ Ulrich Engel, Helmut Schumacher: Kleines Valenzlexikon deutscher Verben, Tübingen ${ }^{2} 1978$.

${ }^{49}$ Ulrich Engel:Die deutschen Satzbaupläne, in: WW 20, 1970, S. 372.

${ }^{50}$ Mit Bezug auf das Beispiel Der Anwalt handelte rasch.

${ }^{51}$ Ebd.

52 Kirsten Adamzik: Ergänzungen zu Ergänzungen und Angaben, in: Deutsche Sprache. Zeitschrift für Theorie, Praxis, Dokumentation 4, 1992, S. 303.

${ }^{53}$ Auf diese Bedingungen gehe ich im Laufe dieser Arbeit ein.
} 
Anhand welcher Kriterien können sie von Angaben unterschieden werden?

\section{Verben im Einzelnen}

Für meine Analyse habe ich die Verben ausgewählt, die am häufigsten in Verbindung mit den genannten Satzgliedern in Wörterbüchern erfasst werden und deren Status als Ergänzung oder Angabe strittig ist. Es handelt sich um die Verben stattfinden, gebären, sterben, geschehen, anfangen, öffnen und beginnen.

\subsection{Stattfinden}

Bei stattfinden habe ich die stärkste Schwankung feststellen können: Die Adverbialbestimmung ist nach Engel/Savin ${ }^{54}$ eine obligatorische Ergänzung, nach Engel/Schumacher ${ }^{55}$ eine fakultative Ergänzung und nach Schumacher ${ }^{56}$ eine Angabe (bei Helbig/Schenkel ${ }^{57}$ kommt das Verb leider nicht vor). In VALBU ${ }^{58}$ wird eine örtliche und zeitliche Adverbativergänzung aufgeführt.

300 Belege sind von mir untersucht worden. Wie man den Ergebnissen entnehmen kann, ist das Vorkommenshäufigkeitsprinzip erfüllt. ${ }^{59}$

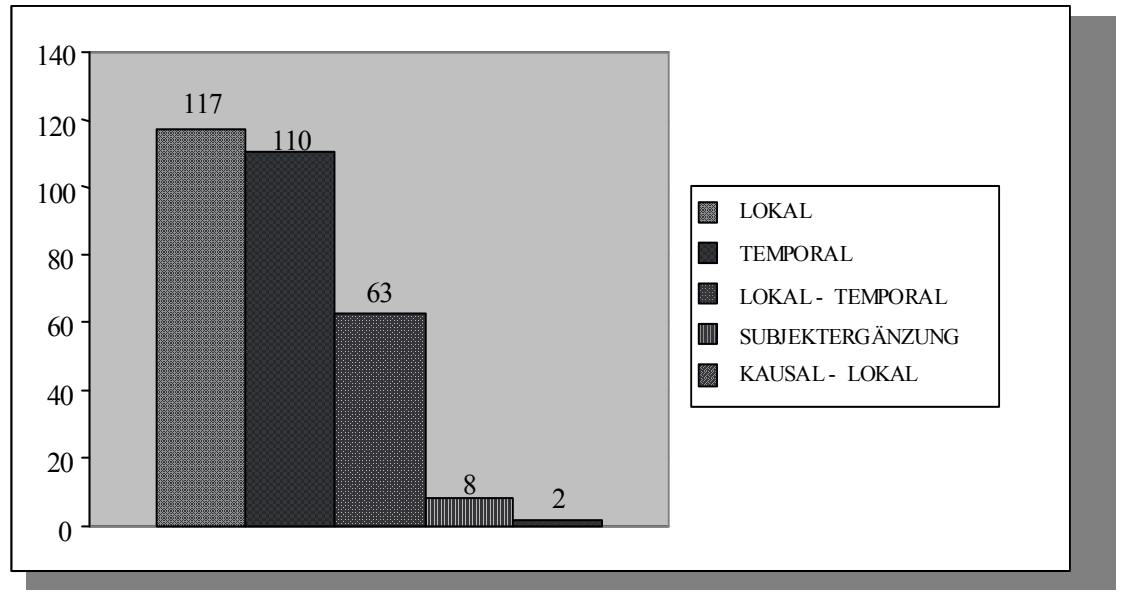

Wie man der Tabelle entnehmen kann, kommen in 117 Belegen eine Lokalergänzung, in 110 eine Temporalergänzung, in 63 sowohl eine Lokal- als auch eine Temporalergänzung und in 2 Belegen sowohl eine Kausal- als auch eine Lokalergänzung vor. Nur in 8 Belegen tritt allein der Satzbauplan Subjektergänzung auf.

\footnotetext{
${ }^{54}$ Engel, Savin [Anm. 5].

${ }^{55}$ Engel, Schumacher [Anm. 48], S. 31.

${ }^{56}$ Schumacher [Anm. 12].

${ }^{57}$ Helbig, Schenkel [Anm. 24].

${ }^{58}$ Schumacher u.a. [Anm. 2].

${ }^{59}$ Bei allen Verben, bei denen temporale, lokale, kausale oder modale Satzglieder das Vorfeld besetzen, habe ich auf Vollständigkeit abgezielt. Wenn diese Satzglieder im Mittelfeld stehen, präsentiere ich nur eine repräsentative Sammlung.
} 
Folgende Positionen sind bei Hauptsätzen zu beobachten:

\begin{tabular}{|c|c|}
\hline Lokal im Mittelfeld & $\begin{array}{l}\text { „Der Vortrag findet im Geographischen Institut der Universität in L 9, 1-2, Hörsaal 13, } \\
\text { statt.“ (MM Mai, 1989) }\end{array}$ \\
\hline Temporal im Mittelfeld & „Die Besichtigung findet am Donnerstag, 3. August, 14.30 Uhr, statt.“ (MM Juli, 1989) \\
\hline $\begin{array}{l}\text { Temporal im Mittelfeld } \\
\text { Lokal im Mittelfeld }\end{array}$ & $\begin{array}{l}\text { „Der Ostermarkt in Oppau findet vom Samstag, 25. März, bis Montag, 28. März, auf } \\
\text { dem Platz an der August-Bebel-Straße statt.“ (MM März, 1989) }\end{array}$ \\
\hline Temporal im Vorfeld & $\begin{array}{l}\text { „Am Samstag, } 17 \text { Uhr, findet mit dem Kindertheater Hartmannstraße ein Martinsumzug } \\
\text { statt.“(MM November, 1989) }\end{array}$ \\
\hline $\begin{array}{l}\text { Temporal im Vorfeld } \\
\text { Lokal im Mittelfeld }\end{array}$ & „1889 fand in Paris die Weltausstellung statt.“ (MM Januar, 1989) \\
\hline $\begin{array}{l}\text { Lokal im Vorfeld } \\
\text { Temporal im Mittelfeld }\end{array}$ & $\begin{array}{l}\text { „Im Gutenberg-Keller in } N \text { 3, } 11 \text { in der Kunststraße fand vor Jahren die allererste } \\
\text { Lesung statt, der damals auch der hochbetagte Autor Franz Schmitt beiwohnte." (MM } \\
\text { Juni, 1991) }\end{array}$ \\
\hline $\begin{array}{l}\text { Temporal im Vorfeld } \\
\text { Temporal im Mittelfeld } \\
\text { Lokal im Mittelfeld }\end{array}$ & $\begin{array}{l}\text { „Am Samstag, 2. September, findet ab } 7 \text { Uhr auf dem Vorplatz und Sporthof des Hauses } \\
\text { der Jugend wieder ein Flohmarkt für Privattrödler statt.“ (MM August, 1989) }\end{array}$ \\
\hline
\end{tabular}

Aus der Beispielsammlung können folgende Schlussfolgerungen gezogen werden:

口 Vorkommenshäufigkeitsprinzip $\neg$ hohe Anzahl an temporalen und lokalen Satzgliedern. ${ }^{61}$

- Topologie $\rightarrow$ Wenn nur ein temporales, lokales und kausales Satzglied im Satz steht, besteht die Tendenz, dass dieses Glied im Mittelfeld vorkommt. Wenn aber mehr als ein Satzglied dieser Art (temporal, kausal, lokal) auftritt, kann der Satz unterschiedlich organisiert werden. Entweder kommen die temporalen, lokalen und kausalen Satzglieder aneinander gereiht im Mittelfeld vor, wobei die Reihenfolge temporal und lokal ist, oder eins von diesen Satzgliedern wird das Vorfeld besetzen. In diesem zweiten Fall ergibt sich die Tendenz, dass temporale Satzglieder im Vorfeld und lokale Satzglieder im Mittelfeld platziert werden. Bei ganz wenigen Fällen wird das Lokalsatzglied dem Vorfeld vorangestellt. Die Satzgliedstellung ist wesentlich anders als in anderen Beispielen, wie bei den nächsten untersuchten Verben zu erkennen ist.

\subsection{Gebären}

300 Belege sind für die Untersuchung dieses Verbs vorhanden ${ }^{62}$.

${ }^{60} \mathrm{MM}$ ist die Abkürzung für „Mannheimer Morgen“.

${ }^{61} \mathrm{Vgl}$. die Ereignisse bei den Verben anfangen und beginnen 


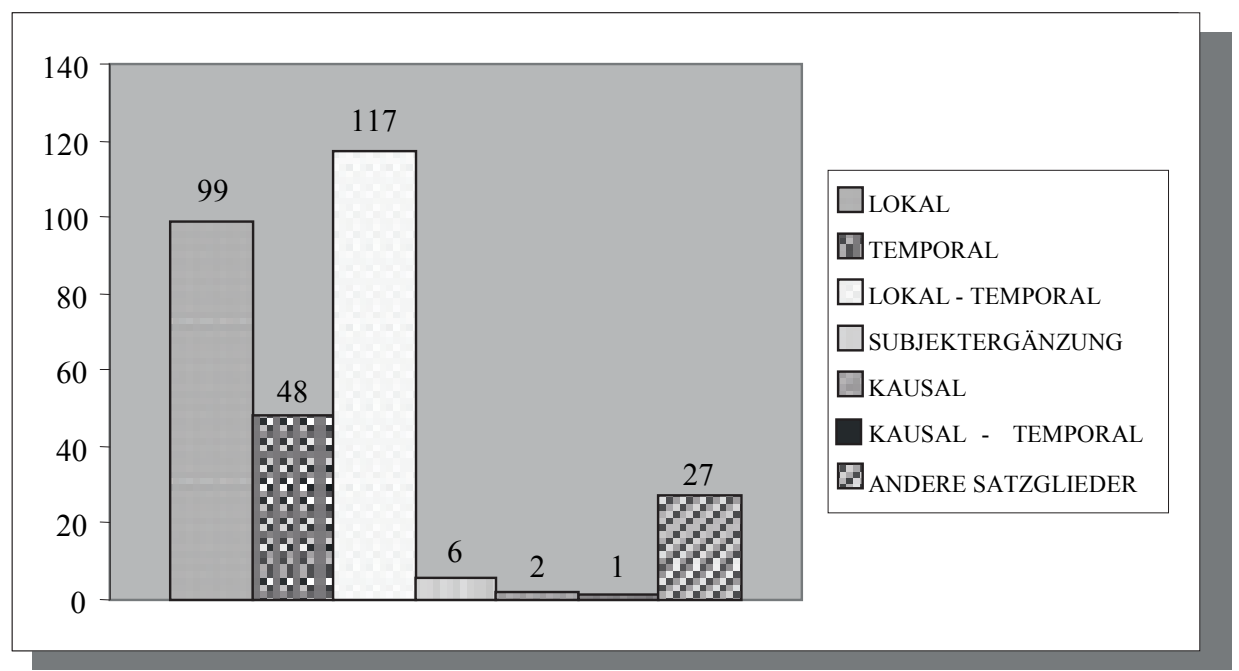

Diese Satzglieder treten im Mittel- und Vorfeld wie folgt auf:

\begin{tabular}{||l||l||}
\hline \hline \begin{tabular}{l} 
Temporal im Mittelfeld \\
\hline Lokal im Mittelfeld
\end{tabular} & „Der Rittersmann wurde 1418 geboren.“(MM Dezember, 1989) \\
\hline $\begin{array}{l}\text { Kausal im Mittelfeld } \\
\text { Temporal im Mittelfeld }\end{array}$ & „Die Verbindung sei zwar aus der Not geboren.“ (MM Oktober, 1994) \\
Lokal im Mittelfeld & „Dr. Ursula Weiss ist 1949 in Mannheim geboren.“ (MM Oktober, 1989) \\
\hline $\begin{array}{l}\text { Lokal im Vorfeld } \\
\text { Temporal im Mittelfeld }\end{array}$ & „Hier wurde er am 9. November 1960 geboren.“ (MM Oktober, 1989) \\
\hline $\begin{array}{l}\text { Prädikativ im Mittelfeld } \\
\text { Lokal im Mittelfeld }\end{array}$ & „Sie wurde als Doris Taylor in Persien geboren.“ (MM Oktober, 1989) \\
\hline
\end{tabular}

- Topologie: Bei den temporalen, kausalen und lokalen Ausdrücken lässt sich bei der Satzgliedstellung eine überwiegende Tendenz zur Besetzung des Mittelfelds feststellen, genauso wie beim vorhergehenden Verb stattfinden. Ausnahmsweise wird in 5 von 300 Belegen das Vorfeld besetzt: „Vor gut zwei Jahren wurde sie geboren.“ (MM März, 1989); „In Seckenheim wurde Werner Seitz geboren.“ (MM September, 1994); „1974 wird Tochter Eva geboren.“ (MM November, 1994); „In Bremen wurde die Schauspielerin geboren.“ (MM September, 1995); „Hier wurde Harry geboren.“ (MM Oktober, 1995).

๑ Unterschiedliche Valenzrealisierungen:

A. ,Man hat etwas geboren', jmd. bringt jmdn. zur Welt'. Transitiver Gebrauch Bsp.: Zwei Töchter hat die Jubilarin geboren (Vorarlberger Nachrichten, 1998)

\footnotetext{
${ }^{62}$ Unter "andere Satzglieder" habe ich im Schema Belege zusammengefasst, bei denen temporale, kausale, lokale und modale Satzglieder mit Ergänzungen wie Akkusativergänzung, Präpositivergänzung usw. vorkommen.
} 


\begin{tabular}{|c|c||c||c||}
\hline \multicolumn{4}{|c|}{ Subjekt } \\
\hline \hline Relationale Bedeutung & Kategorielle Bedeutung & bestimmt & bekannt \\
\hline Agens-Rolle & {$\left[\right.$ hum] $/[\text { [ool }]^{63}$} & + & + \\
\hline
\end{tabular}

In diesem Fall sinkt die Anzahl von temporalen, lokalen und kausalen Satzgliedern ab. Das Agens ist in der Regel die bekannte Information (in der Regel bestimmt) und der Affektiv ${ }^{64}$ bildet in der Regel die neue Information, ist also unbestimmt.

B. ,Etwas geschieht tatsächlich ${ }^{e}$, etwas wird geschehen', ,es gibt ${ }^{e}$, ,entstehen ${ }^{e}$. Absolute Bedeutung.

Bsp.: „Ein neues Polen werde geboren.“ (MM Juli, 1989); „Es war 1926... Ingeborg Bachmann wird geboren.“ (MM Dezember, 1989); „,Fidelio' ist geboren.“ (MM Juli, 1995); „Ein Mythos war geboren und Darrow starb 1967.“(MM August, 1995).

\begin{tabular}{|c||c||c|c||}
\hline \multicolumn{3}{|c||}{ Subjekt } \\
\hline \hline Relationale Bedeutung & Kategorielle Bedeutung & bestimmt & bekannt \\
\hline Nicht-Agens-Rolle & {$\left[\right.$ hum] $/\left[\right.$ zool] $/\left[\right.$ intell] $/\left[\right.$ stat $^{65}$} & - & - \\
\hline
\end{tabular}

Unter dem relationalen Merkmal Nicht-Agens-Rolle kann auch eine andere Gruppe von Beispielen zusammengefasst werden, die durch die Merkmale +bekannt und +bestimmt zu kennzeichnen sind, wie beim Beispiel Das Kind ist geboren - Das Kind ist gerade/tatsächlich geboren $^{66}$. Diese Realisierung, wie man der Graphik entnehmen kann, weist eine hohe Anzahl von temporalen, kausalen, modalen und lokalen Satzgliedern auf.

\section{Wann, wo ist etwas geboren}

Bsp: „Er ist in Mannheim geboren.“ (MM Januar, 1991). Diese Belege bilden die Mehrheit der Sammlung. Bei dieser Valenzrealisierung wird nicht ausgedrückt, ob ,etwas tatsächlich geschieht ${ }^{e}$, sondern ,unter welchen Umständen ${ }^{e}$ etwas geschieht.

Allgemein lässt sich feststellen, dass die temporalen, kausalen und lokalen Satzglieder in höherer Anzahl mit der Nicht-Agens-Rolle und mit den Verbformen Perfekt und Plusquamperfekt vorkommen.

\subsection{Sterben}

Bei diesem Verb sieht man bei den 300 untersuchten Belegen, dass bei mehr als der Hälfte ein lokales oder temporales Satzglied oder beide Satzglieder vorkommen ${ }^{67}$ :

\footnotetext{
${ }^{63}$ [zool] steht für ,Tiere (vgl. Engel [Anm. 5.], S. 358 f.).

${ }^{64}$ Vgl. Ulrich Engel: Semantische Relatoren. Ein Entwurf für künftige Valenzwörterbücher, in: Semantik, Lexikographie und Computeranwendung, hg. v. Nico Weber, Tübingen 1996, S. 223-236.

${ }^{65}$ [intell] steht für ,geistige (vgl. Engel [Anm. 5.], S. 358 f.).

${ }^{66}$ Denken wir an die Verwandten, die in dem Warteraum eines Krankenhauses sitzen. Der Arzt kommt raus und sagt: „Das Kind ist geboren“.

${ }^{67}$ Unter ,andere Satzglieder“ habe ich im Schema Belege zusammengefasst, bei denen temporale, kausale, lokale und modale Satzglieder mit Ergänzungen wie Akkusativergänzung, Präpositivergänzung usw. vorkommen.
} 


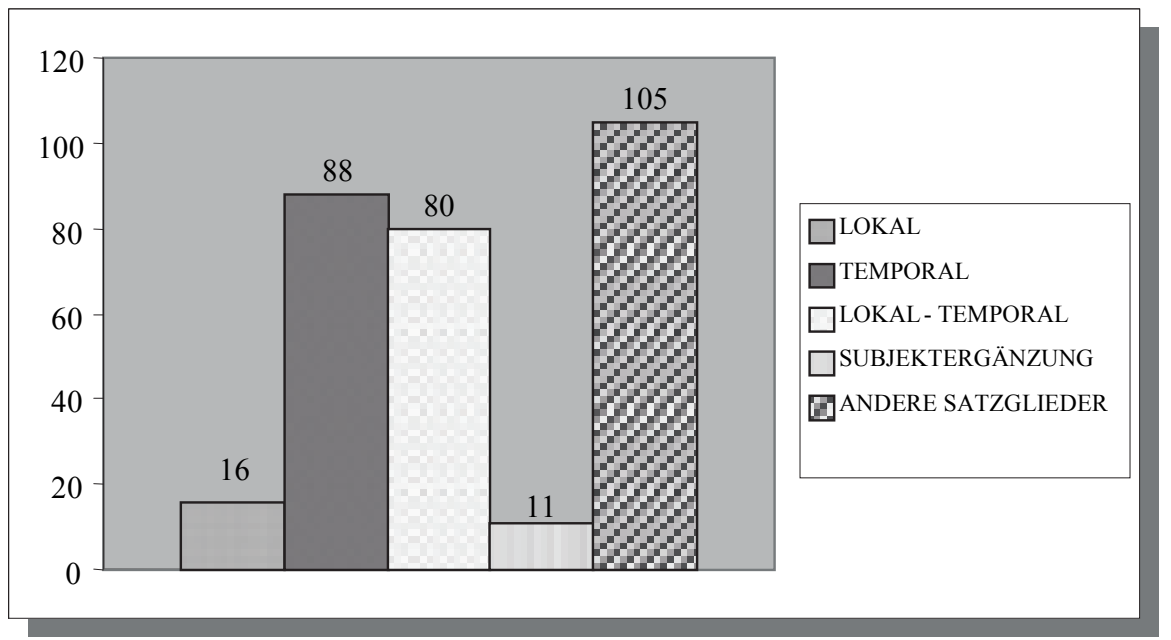

Die Topologie der Satzglieder:

\begin{tabular}{|c|c|}
\hline Temporal im Mittelfeld & „Er starb am 26. Juni 1914.“ (MM Juni, 1989) \\
\hline Lokal im Mittelfeld & „Sie starb 81jährig in ihrem Haus in Cornwall.“ (MM April, 1989) \\
\hline $\begin{array}{l}\text { Lokal im Mittelfeld } \\
\text { Präpositivergänzung im Mittelfeld }\end{array}$ & $\begin{array}{l}\text { „Der Mann starb an der Unfallstelle an den Folgen der Verletzungen.“ (MM } \\
\text { April, 1989) }\end{array}$ \\
\hline $\begin{array}{l}\text { Temporal im Mittelfeld } \\
\text { Lokal im Mittelfeld } \\
\text { Präpositivergänzung im Mittelfeld }\end{array}$ & $\begin{array}{l}\text { „Doch der österreichische Maler, Grafiker und Illustrator starb bereits am } \\
\text { 21. März } 1988 \text { in Mödling bei Wien. "(MM Januar, 1989); „Drei Tage nach } \\
\text { seinem } 58 . \text { Geburtstag ist der Schriftsteller Thomas Bernhard in Gmunden } \\
\text { (Oberösterreich) an Herzversagen gestorben. “(MM Februar, 1989) }\end{array}$ \\
\hline Temporal im Vorfeld & $\begin{array}{l}\text { „Am Sonntag starb, wie erst jetzt bekannt wurde, der österreichische Autor } \\
\text { Thomas Bernhard. (MM Februar, 1989) }\end{array}$ \\
\hline Lokal im Vorfeld & $\begin{array}{l}\text { „In seiner Heimatstadt Köln starb der 83jährige Willy Schneider.“ (MM } \\
\text { Januar, 1989) }\end{array}$ \\
\hline $\begin{array}{l}\text { Lokal im Vorfeld } \\
\text { Temporal im Mittelfeld }\end{array}$ & $\begin{array}{l}\text { „Noch am Unfallort starb am Mittwochnachmittag ein Radfahrer." (MM } \\
\text { Mai, 1989) }\end{array}$ \\
\hline $\begin{array}{l}\text { Temporal im Vorfeld } \\
\text { Temporal im Mittelfeld }\end{array}$ & „Aber dann starb 1962 der Vater.“ (MM März, 1989) \\
\hline $\begin{array}{l}\text { Temporal im Vorfeld } \\
\text { Lokal im Mittelfeld } \\
\text { Präpositivergänzung im Mittelfeld }\end{array}$ & $\begin{array}{l}\text { „Am 29. April } 1951 \text { starb er in Cambridge.“ (MM April, 1989); „Noch vor } \\
\text { Eintreffen des Notarztes starb eine 23jährige Frau an den Verletzungen.“ } \\
\text { (MM Juli, 1989) }\end{array}$ \\
\hline
\end{tabular}

a Bezüglich der Topologie tendiert man dazu, die temporalen und lokalen Satzglieder ins Mittelfeld zu stellen. In den Fällen, in denen sie eine andere Stelle besetzen, kann man Folgendes beobachten: Entweder steht eins von diesen Satzgliedern im Vorfeld, wenn im 
Mittelfeld ein anderes dieser Satzglieder steht, oder eins von diesen Satzgliedern steht in sehr wenigen Fällen im Vorfeld. ${ }^{68}$

a Für die Valenzrealisierung scheinen hier die Verbtempora entscheidend zu sein. Die Verbformen dieser 300 Belege stehen in der Regel im Präteritum oder im Perfekt. Für mich stellt sich die Frage, welche Rolle das Vorkommen von Präteritum und Perfekt für die Valenzrealisierung spielt. Unter den 300 untersuchten Belegen tritt der Satzbauplan Subjektergänzung nur $11 \mathrm{Mal}$ auf, und dieser in Belegen, die im Präsens stehen. Um eine mögliche Korrelation zwischen dem Verbtempus und dem Satzbauplan festzustellen, habe ich mehr als einhundert Belege im Präsens nachgeschlagen. Das Ergebnis ist eindeutig: Beim Präsens steht am häufigsten der Satzbauplan Subjektergänzung. Meiner Ansicht nach bedeutet dies, dass das Verb sterben mehr als eine Valenzrealisierung hat, d.h. im Präteritum und Perfekt werden temporale und lokale Satzglieder erwartet, im Präsens kommen sie nicht vor, es handelt sich um eine absolute Bedeutung. Da mit den unterschiedlichen Valenzrealisierungen unterschiedliche Bedeutungen hervorgehen, sollte dies meiner Ansicht nach in Form unterschiedlicher Lexikoneinträge berücksichtigt werden. Diese absolute Bedeutung sieht man auch bei dem Modus Verbi Imperativ, was ich wie folgt belege: „Hilf dir selbst oder stirb.“ (MM September, 1995); „Mach es oder stirb“ (Frankfurter Rundschau April, 1997); „Lauf oder stirb“ (Frankfurter Rundschau April, 1998); „Flieg weiter, Vogel oder stirb!“" (MM Mai, 1998); „Go or death! -schaff es oder stirb!“ (Frankfurter Rundschau Juli, 1999); „Sex oder stirb!“" (MM November, 2000).

\subsection{Geschehen}

Nach der Untersuchung von 300 Belegen bin ich zu folgendem Ergebnis gekommen ${ }^{69}$ :

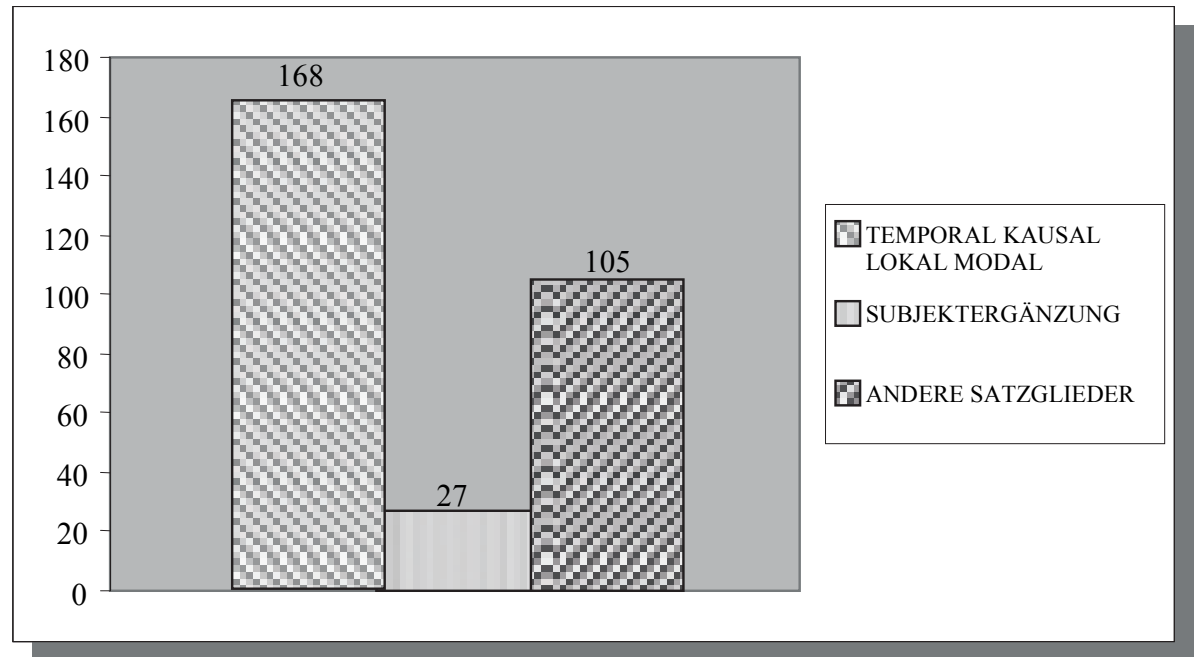

${ }^{68}$ In diesem Fall sollte man thematische und rhematische Aspekte nicht vernachlässigen. Auf dieses Thema greife ich im Abschnitt 4. Analyse der Ergebnisse zurück.

${ }^{69}$ Satzmuster wie mit etw./jmdm. geschehen und jmdm. geschehen sind in der Tabelle unter ,andere Satzglieder“ zusammengefasst worden. Unter ,andere Satzglieder“ habe ich im Schema Belege zusammengefasst, bei denen temporale, kausale, lokale und modale Satzglieder mit Ergänzungen wie Akkusativergänzung, Präpositivergänzung usw. vorkommen. 
Die Topologie der Satzglieder:

\begin{tabular}{||l||l||}
\hline \hline Temporal im Mittelfeld & „Dies geschieht am Montag, 12. Juni, um 13.30.“ (MM Juni, 1989) \\
\hline Lokal im Mittelfeld & „Das geschieht in Südamerika, es geschieht auf den Philippinen.“ (MM Mai, 1989) \\
\hline Modal im Mittelfeld & „Das geschieht alles zielbewußt und mit Witz.“ (MM Juli, 1989); “Dies geschieht \\
pragmatisch und ohne Eile.” (MM Juli, 1991)
\end{tabular}

Im Gegensatz zu den bisher untersuchten Verben kann man bei geschehen eine höhere Anzahl von modalen und kausalen Satzgliedern beobachten. Wenn nur eine dieser Satzgliedklassen vorkommt, steht sie meist im Mittelfeld. Wenn mehrere Satzglieder dieser Art vorkommen, werden sowohl das Vorfeld als auch das Mittelfed besetzt. In diesem Fall steht häufig das temporale Satzglied im Vorfeld.

Im Weiteren möchte ich auf Belege mit dem Satzbauplan Subjektergänzung (nur 27 Belege insgesamt) eingehen:

a. Als Nebensatz

Durch konditionalen Nebensatz: „Aus den spärlichen Spuren, die der Medikamenten-Cocktail im Körper des CDU-Politikers hinterlassen hat, läßt sich, wenn kein Wunder geschieht, das Ende nicht mehr mit letzter Gewißheit rekonstruieren." (MM August, 1995)

Als expansiver Nebensatz: „Die Leute tun oftmals sagen, du hättest eine andere Lieb, doch glaub ich es nicht, bis es geschieht..." (MM November, 1989)

Als Akkusativergänzung: „Sie bestimmen eben selbst, was geschieht.“ (MM März, 1989)

Als Präpositivergänzung mit oder ohne Korrelat: „Der Nachfrage-Stau würde in einer Marktwirtschaft zu steigenden Preisen führen; in der DDR-Planwirtschaft sorgen jedoch staatlich festgelegte Preise dafür, daß dies nicht geschieht.“ (MM Dezember, 1989)

b. Als Hauptsatz

Subjektergänzung: „Es geschieht nichts.“ (MM März, 1989) „Nichts geschieht, und doch schauen wir gebannt.“(MM Oktober, 1989) „Unvernünftiges geschieht, Sinnloses.“ (MM Mai, 1991)

Durch wenn-Nebensatz erweitert: „Was geschieht eigentlich, wenn wir morgens erwachen?“ (MM Januar, 1989) 
Meiner Auffassung nach können folgende Valenzrealisierungen unterschieden werden:

1. Ein Unfall ist geschehen

\begin{tabular}{|c||c|c|c|}
\hline \multicolumn{3}{|c|}{ Subjekt } \\
\hline \hline Relationale Bedeutung & Kategorielle Bedeutung & bestimmt & bekannt \\
\hline Nicht-Agens-Rolle & {$[\mathrm{akt}]$} & - & - \\
\hline
\end{tabular}

Dieser Ausdruck ist kommunikationsangemessen. Es kann die Situation auftreten, dass der Sprecher auf die Frage Was ist geschehen? antworten muss.

2. Der Unfall geschah

\begin{tabular}{|c||c|c|c|}
\hline \multicolumn{3}{|c|}{ Subjekt } \\
\hline \hline Relationale Bedeutung & Kategorielle Bedeutung & bestimmt & bekannt \\
\hline Nicht-Agens-Rolle & {$[$ akt] } & + & + \\
\hline
\end{tabular}

Man kann sich eine solche Äußerung nur vorstellen, wenn jemand sich vergewissern möchte, ob der Unfall geschehen ist, sonst mangelt es der Äußerung an Informationsgehalt und dem Ausdruck an Grammatikalität.

3. Der Unfall geschah gestern/aus Versehen/auf der Straße

\begin{tabular}{|c|c|c|c|}
\hline \multicolumn{4}{|c|}{ Subjekt } \\
\hline Relationale Bedeutung & Kategorielle Bedeutung & bestimmt & bekannt \\
\hline Nicht-Agens-Rolle & [akt] & + & + \\
\hline
\end{tabular}

Bei diesem Beispiel, in dem temporale, lokale usw. Satzglieder vorkommen, kann man sich einen Sprecher vorstellen, der denkt, dass der Hörer schon weiß, dass der Unfall geschehen ist, und in diesem Fall möchte der Sprecher eine bestimmte für den Hörer unbekannte Information übertragen.

In welchem Zusammenhang stehen die Subjektergänzung und das Vorkommen von temporalen, lokalen oder kausalen Satzgliedern? Zur Antwort werden folgende Belege analysiert:

1. „Alle 19 Jahre geschieht dies.“" (MM Juni, 1995)

2. „Derzeit geschieht dies für Arzt- und Tierarzthelferinnen, ab 1990/91 folgen die Industrieberufe im Bereich Metalltechnik.“(MM Juli, 1989)

3. „Seit Jahren aber geschieht von staatlicher Seite fast nichts.“ (MM November, 1989)

4. „Dann aber geschieht etwas völlig Unerwartetes.“ (MM März, 1989)

5. “Als sie an einem Sonntag länger schläft, geschieht ein Unglück (ARD: 20.15 Uhr).“(MM April, 1989)

6. "Dann geschieht ein zweiter Mord, in den Rosa (...).“ (MM November, 1994)

7. „Zur selben Zeit geschieht ein Mord.“ (MM Oktober, 1995) 
8. ,Erst auf Seite 187 geschieht ein Mord.“ (MM April, 1991)

9. „Immer häufiger geschieht es, daß Klemens Müller Briefe...“(MM Januar, 1991) ${ }^{70}$

10. „Nicht selten geschieht es, daß an einem Wochenendseminar...“(MM September, 1995)

Wie den Belegen entnommen werden kann, kann die Vorfeld- oder Mittelfeldbesetzung durch kommunikative Parameter erklärt werden, was an bestimmten morphosyntaktischen Merkmalen bei diesen Äußerungen widergespiegelt wird. Zur Veranschaulichung dient exemplarisch der Beleg 7. „Zur selben Zeit geschieht ein Mord.“ (MM Oktober, 1995):

Ein Mord ist die Subjektergänzug, relational kann sie als Nicht-Agens und kategoriell als [akt] beschrieben werden. Sie ist auch -bestimmt und dem Hörer nicht bekannt. Der Satz ist grammatisch und kommunikationsangemessen. Der Fragetest lautet: Was ist geschehen? Weil „ein Mord“ das Rhema ${ }^{71}$ ist, wird diese Nominalphrase ins Mittelfeld gestellt. Im Vorfeld steht das temporale Satzglied, das der bekannten Information entspricht. Eine andere Valenzrealisierung liegt dagegen beim Beispiel Der Mord geschieht zur selben Zeit vor: erstens lautet der Fragetest Wann geschieht der Mord? Zur selben Zeit; zweitens ergibt sich mittels des Reduktionstests eine andere Bedeutung als die von Der Mord geschieht zur selben Zeit, nämlich der Mord geschieht, wobei der Hörer und Sprecher wissen muss, um welchen Mord es sich handelt. Es ist die bekannte Information. Der Fragetest lautet Geschieht tatsächlich der Mord?. Aus dem bereits Genannten plädiere ich dafür, dass bei Der Mord geschieht zur selben Zeit eine Temporalergänzung vorkommt.

Es stellt sich hier auch die Frage, ob das bereits Genannte vom Verbtempus bedingt wird. Meines Erachtens ist dies nicht davon abhängig, da durch die Anwendung des Fragetests bei den Beispielen ein Mord geschieht und der Mord ist geschehen gleiche Ergebnisse hervorgehen. Im ersten Fall lautet die Frage „Was geschieht?“ und im zweiten „Ist der Mord wirklich geschehen?". In der Regel treten temporale, kausale und weitere Satzglieder im zweiten Fall häufiger auf, wo die Subjektergänzung als + bestimmt und + bekannt beschrieben werden kann. In diesem Fall kann man an der Mehrheit der Korpusbelege beobachten, dass die Subjektergänzung, der Mord, dazu neigt, im Vorfeld zu stehen.

\subsection{Anfangen. Öffnen. Beginnen}

Die drei Verben anfangen, beginnen und öffnen werde ich im Sinne eines Vergleichs anführen, denn diese Verben zeigen sehr deutlich eine transitive und eine intransitive Realisierung. Wie ich erläutert habe, werde ich aufgrund der unterschiedlichen Realisierungen dieser Verben den Versuch unternehmen, mögliche topologische Unterschiede festzustellen. Deshalb werde ich die Verben anfangen, öffnen und beginnen intensiver analysieren.

\footnotetext{
${ }^{70}$ Der Subjunktorsatz (dass) erfüllt die Funktion der Subjektergänzung. Es ist ein Korrelat. Die gleiche Erklärung gilt für Beispiel 10.

71 Genauso bei Beispiel 1. und 4. kann die Vorfeldbesetzung durch die temporalen Satzglieder nach Gliederungsprinzipien wie Thema und Rhema erklärt werden.
} 


\section{A. Anfangen}

Bei den untersuchten Belegen können zwei Bedeutungsrealisierungen differenziert werden:

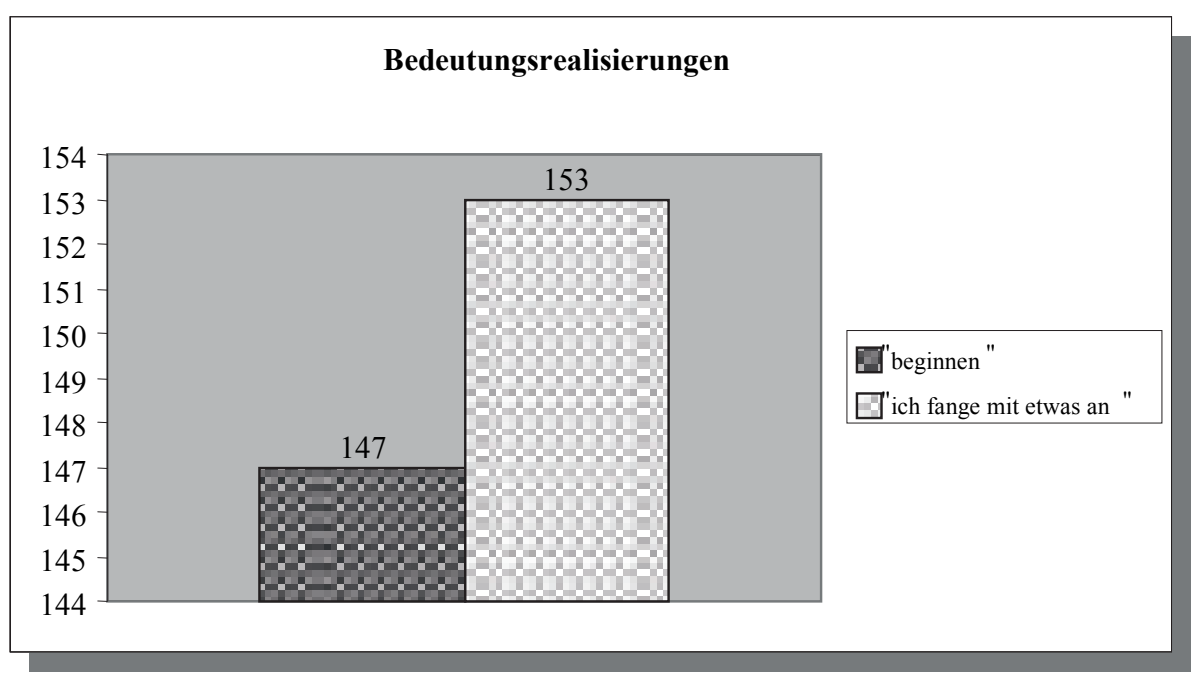

A.1. Intransitive Valenzrealisierung. Im Sinne von ,beginnen', ,etwas fängt an ${ }^{`}$. Es kommen 147 Belege vor, wobei nur in einem einzigen Fall der Satzbauplan Subjektergänzung steht. Bei dieser Realisierung ergibt sich die höchste Häufigkeitszahl beim Vorkommen von temporalen, lokalen u.a. Satzgliedern. In einer geringeren Anzahl kommen temporale, lokale Satzglieder bei der zweiten Gruppe (A.2.) vor.

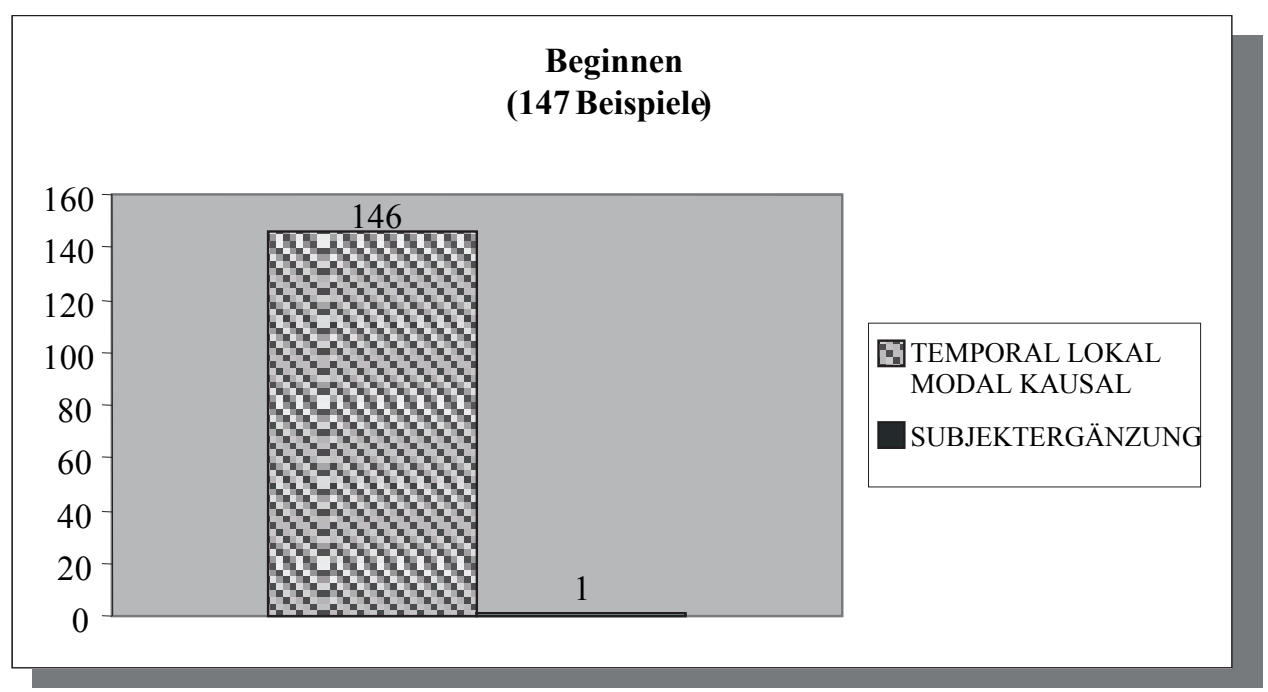


A.2. Präpositionale und transitive Valenzrealisierung. Im Sinne von, ich fange mit etwas an ${ }^{e}$ und ,ich fange etwas an ${ }^{72}$

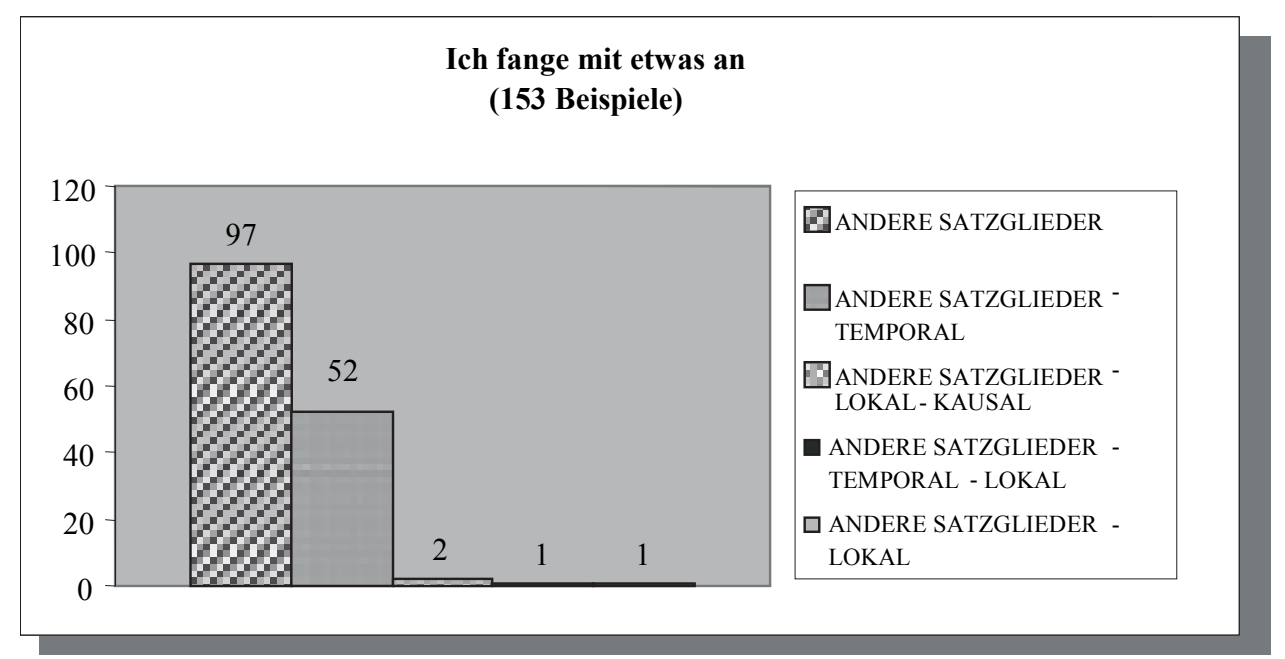

- Satzkonstruktion ,etwas fängt an`. Gruppe 1. Ergänzungskandidaten?

\begin{tabular}{|c|c|}
\hline Lokal im Mittelfeld & „Schulferien fangen außerdem in Dänemark an.“ (MM März, 1989) \\
\hline Modal im Mittelfeld & „Wir fangen nicht neu an.“( (MM März, 1989) \\
\hline $\begin{array}{l}\text { Temporal im Mittelfeld } \\
\text { Kausal im Mittelfeld }\end{array}$ & $\begin{array}{l}\text { „Wir fangen in diesem Jahr durch die Einteilung in Sparten praktisch neu an.“ (MM } \\
\text { Januar, 1996) }\end{array}$ \\
\hline $\begin{array}{l}\text { Lokal im Vorfeld } \\
\text { Temporal im Mittelfeld }\end{array}$ & $\begin{array}{l}\text { „Bei uns fangen jetzt mindestens zwei russische Studenten an.“ (MM September, 1994); } \\
\text { „Doch hier fangen die Probleme erst an.“(MM Dezember, 1997) }\end{array}$ \\
\hline $\begin{array}{l}\text { Temporal im Vorfeld } \\
\text { Modal im Mittelfeld }\end{array}$ & $\begin{array}{l}\text { „1949 mußte er wieder ganz von vorne anfangen.“ (MM Juni, 1996),“Aber am Sonntag } \\
\text { werden wir von vorne anfangen.“ (MM April, 1998) }\end{array}$ \\
\hline $\begin{array}{l}\text { Kausal im Vorfeld } \\
\text { Temporal im Mittelfeld }\end{array}$ & „Deswegen fangen sie früher an.“(MM Mai, 1999) \\
\hline Temporal im Vorfeld & $\begin{array}{l}\text { „,Ab Montag fangen die Detailplanungen und die Vermarktung an', erklärte der } \\
\text { Bauunternehmer."(MM Februar, 1998) }\end{array}$ \\
\hline Lokal im Vorfeld & „Bei euch zu Hause fangen möglicherweise die Probleme an.“(MM Oktober, 1999) \\
\hline
\end{tabular}

- Satzkonstruktion ,jemand fängt mit etwas an`. Gruppe 2. Angabekandidaten?

\begin{tabular}{||l||l||}
\hline \hline Temporal im Vorfeld & $\begin{array}{l}\text { „Jetzt fangen sie an zu spinnen.“ (MM Oktober, 1994) ,Ja, super, am 1. September fang } \\
\text { ich an. “(MM Juli, 1998) }\end{array}$ \\
\hline Lokal im Vorfeld & $\begin{array}{l}\text { „In Deutschland, so Diplomingenieur Schwanhold, hätte Bill Gates nie in einer Garage } \\
\text { anfangen dürfen.“ (MM November, 1995) }\end{array}$ \\
\hline
\end{tabular}

${ }^{72}$ Bei etwas/nichts mit etw. anfangen habe ich keine temporalen, kausalen oder lokalen Satzglieder in der Beispielsammlung gefunden. 


\begin{tabular}{|c|c|}
\hline Temporal im Mittelfeld & „Sie fangen erst um 9 Uhr vormittags mit der Arbeit an.“ (MM November, 1995). \\
\hline $\begin{array}{l}\text { Temporal im Vorfeld } \\
\text { Lokal im Mittelfeld }\end{array}$ & $\begin{array}{l}\text { „Jetzt fangen wir wieder bei Null an." (MM März, 1989),“Jetzt müssen wir unten } \\
\text { anfangen." (MM Juni, 1989) }\end{array}$ \\
\hline $\begin{array}{l}\text { Temporal im Mittelfeld } \\
\text { Lokal im Mittelfeld }\end{array}$ & $\begin{array}{l}\text { „Die Herrenmannschaft muß dieses Jahr ganz von vorne anfangen und wieder in der } \\
\text { Bezirksklasse } 2 \text { starten, da letztes Jahr keine Mannschaft gemeldet werden konnte.“ } \\
\text { (MM Januar, 1998) }\end{array}$ \\
\hline $\begin{array}{l}\text { Temporal im Mittelfeld } \\
\text { Lokal im Mittelfeld } \\
\text { Modal im Mittelfeld }\end{array}$ & $\begin{array}{l}\text { „Ich fange nicht mehr irgendwo neu an." (MM Juli, 1996); „Gleich auf Seite } 1 \text { steht der } \\
\text { Satz ,Viele werden in ihrem Leben noch einmal neu anfangen" -was die Wirklichkeit ist.“ } \\
\text { (MM April, 1998) }\end{array}$ \\
\hline
\end{tabular}

Sowohl in der Gruppe 1. als in der Gruppe 2. schwankt die Topologietendenz. Folgende Valenzrealisierungen können unterschieden werden:

1. etwas beginnt ${ }^{`}$, etwas fängt an`: 147 Belege, nur ein Beleg ohne temporale, lokale Satzglieder.

\begin{tabular}{|c||c||c||c|}
\hline \multicolumn{4}{|c||}{ Subjekt } \\
\hline Relationale Bedeutung & Kategorielle Bedeutung & bestimmt & bekannt \\
\hline Nicht-Agens-Rolle & {$[\mathrm{akt}] /[\mathrm{stat}]$} & $+/-$ & $+/-$ \\
\hline
\end{tabular}

2. ,Ich fange mit etwas an

\begin{tabular}{|c||c|c||c||}
\hline \multicolumn{4}{|c|}{ Subjekt } \\
\hline \hline Relationale Bedeutung & Kategorielle Bedeutung & bestimmt & bekannt \\
\hline Agens-Rolle & {$[\mathrm{hum}] /[\mathrm{inst}]$} & $+/-$ & $+/-$ \\
\hline
\end{tabular}

Die Ergebnisse sind denjenigen des Verbs beginnen ähnlich (siehe 3.5.C.).

\section{B. Öffnen}

Das Verb öffnen lässt sich genauso wie das Verb anfangen erklären. In Bezug auf dieses Verb erweist sich die Untersuchung der Verbtempora als grundlegend. Während bei dem transitiven Gebrauch des Verbs überwiegend das Präsens verwendet wird ${ }^{73}$, wird z.B. beim intransitiven Gebrauch das Perfekt angewendet.

Darüber hinaus scheint das Genus verbi auch eine entscheidende Rolle zu spielen:

a. Aktivform: „die Veterama für die Autoliebhaber öffnet am 22. und 23. April, die für Motorradfans am 29. und 30. April 1995 ihre Pforte“ (MM, September, 1994); „Herr X verreist nach Mailand. Seine Haustür verriegelt er mit einer multifunktionellen Prozessor- Chipkarte, die auch sein Auto öffnet und startet" (MM, November, 1994); „,Dieser ganz besondere Bildungsgang erfordert große Anstrengungen, öffnet aber eine große Zukunftsperspektive', erklärte Edmund Fasnacht, [...]“" (MM, August, 1995)

b. werden- und sein-Passiv-Belege $=$ volles Passiv

\footnotetext{
${ }^{73}$ In dieser Gruppe tritt das Perfekt nur bei 13 Belegen auf.
} 


\section{b.1. Vorgangspassiv}

Ohne temporale Satzglieder. Absoluter Gebrauch: „Nachdem die Bildungstore geöffnet wurden, strömten (...)“(MM April, 1989); „Wird das Ventil geöffnet, preßt die Druckluft den Beutel.“ (MM Mai, 1989); „Selbst der Vorhang wird ,von Hand ' geöffnet.“ (MM Juli, 1989); „Nach Angaben des österreichischen Roten Kreuzes wurden in der Nacht sechs Grenzübergangsstellen von Ungarn nach Österreich geöffnet, an denen die Organisation den DDR-Bürgern auch Hilfe leistet.“ (MM September, 1989); „Ihnen wurde Tür und Tor geöffnet.“(MM November, 1989)

\section{b.2. neutrales Passiv}

Temporal im Vorfeld: „Um halb fünf wurde geöffnet, das Schauspiel begann pünktlich.“ (MM Mai, 1989)

\section{b.3. Zustandspassiv}

Mit temporalen Satzgliedern im Mittelfeld: „Das Festzelt ist ab 8.30 Uhr geöffnet.“ (MM April, 1989); „Der Dom war von 19 Uhr an für Besucher geöffnet.“ (MM Juli, 1989); „Cafes und Restaurants sind durchgehend geöffnet!“ (MM Oktober, 1989); „Die Tageskasse ist von 11 bis 13 und von 16 bis 18 Uhr geöffnet.“ (MM Dezember, 1989); „Die Marionetten-Werkstatt ist am Sonntag ab 14 Uhr geöffnet.“ (MM November, 1989); „Giardini und Correr Museum sind täglich geöffnet außer montags.“ (MM Juni, 1995)

Während bei der Aktivform (a) und bei dem werden-Passiv (Vorgangspassiv, b.1.) temporale Satzglieder nur in 2 Belegen, lokale nur in 3 Belegen vorkommen, stehen beim neutralen Passiv und beim sein-Passiv (Zustandspassiv) oftmals temporale Satzglieder ${ }^{74}$. Bei b.2. und b.3. kommen nur 8 Beispiele ohne temporale Satzglieder vor. Das überwiegende Vorkommen von temporalen Satzgliedern bei der Satzstruktur ,etwas ist geöffnet muss hier hervorgehoben werden, so dass das Erwartbarkeitskriterium erfüllt ist. Im topologischen Bereich ist entscheidend, dass nur in 4 Belegen der ganzen Sammlung das Temporalsatzglied am Satzanfang steht. Im Sinne eines Vergleichs werde ich Belege aus a., b.2. und b.3. zur Betrachtung heranziehen:

Beleg aus a. „Er hat mir das Tor zur Motorsportwelt geöffnet.“ (MM Januar, 1991)

Transitive Valenzrealisierung

Subjekt: Agens-Rolle, +bestimmt, +bekannt.

Fragetest: Was hat er geöffnet?.

Beleg aus b.3. „Das Festzelt ist ab 8.30 Uhr geöffnet“ (MM April, 1989)

sein-Passiv

Subjekt: Nicht-Agens-Rolle, +bekannt, +bestimmt.

Fragetest: Wann ist das Festzelt geöffnet?. Die Frage Ist das Festzelt geöffnet? bezieht sich dagegen auf das Beispiel Das Festzelt ist geöffnet.

Bei den Belegen b.2. („Um halb fünf wurde geöffnet, das Schauspiel begann pünktlich“) lautet die Frage Wann wurde geöffnet?

\footnotetext{
${ }^{74}$ Ein gleiches Vorgehen liegt bei sterben vor.
} 
Bei diesem Verb können aufgrund der Genera Verbi Unterschiede festgestellt werden: Während bei der Aktivform und dem Vorgangspassiv die Anzahl der analysierten Satzglieder niedrig ist, liegt die Anzahl lokaler Satzglieder beim neutralen Passiv und Zustandspassiv höher. Das entspricht wieder den bei den anderen Verben nachgewiesenen Ergebnissen (vgl. A. anfangen und C. beginnen).

\section{Beginnen}

Ich werde ebenso wie bei dem Verb anfangen verfahren, d.h. ich halte es für notwendig, bei der Unterscheidung der Valenzrealisierungen $\mathrm{zu}$ bleiben und $\mathrm{zu}$ untersuchen, wie häufig temporale, lokale, kausale und modale Satzglieder bei der Satzstruktur ,etwas beginnt ${ }^{\circ}$ im Vergleich zu ,ich beginne mit etwas ${ }^{e}$ vorkommen (das Erwartbarkeitsprinzip) und an welcher Stelle diese Satzglieder platziert sind.

Das Vorkommen dieser Glieder wird anhand folgender Graphik dargestellt ${ }^{75}$ :

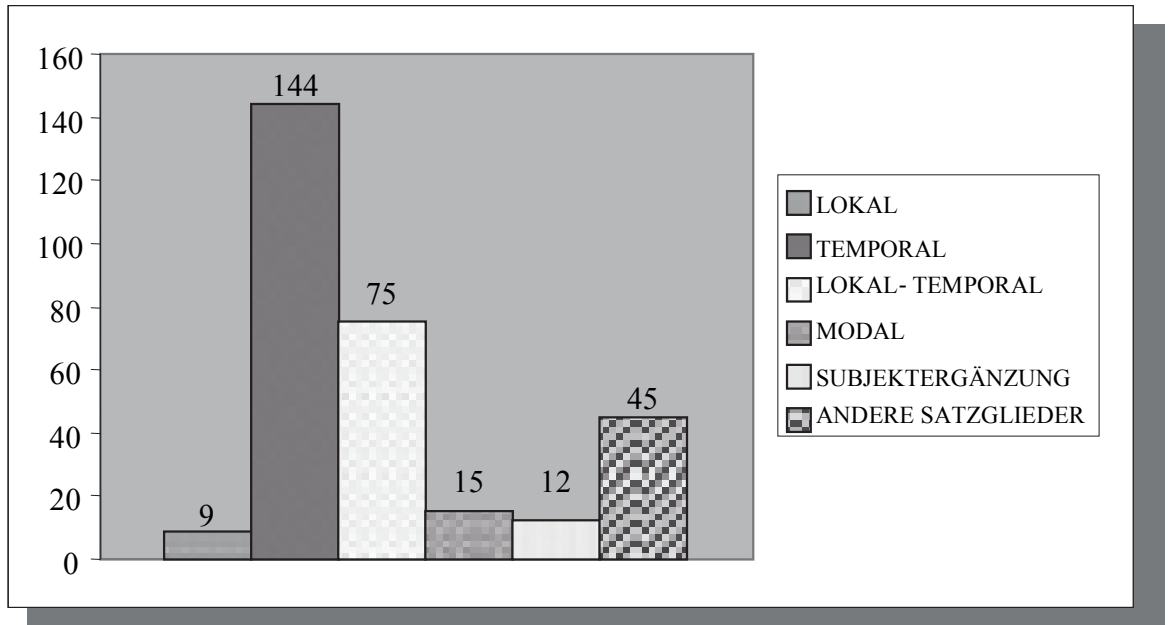

Folgende Positionen sind bei beginnen zu beachten ${ }^{76}$ :

- Satzkonstruktion ,etwas beginnt ${ }^{e}$. Intransitive Valenzrealisierung

\begin{tabular}{|c|c|}
\hline Temporal im Mittelfeld & „Der Kurs beginnt am 2. Februar.“ (MM Januar, 1989) \\
\hline Lokal im Mittelfeld & „Die Arbeit beginnt und endet an der Arbeitsstelle.“ (MM November, 1994) \\
\hline $\begin{array}{l}\text { Lokal im Mittelfeld } \\
\text { Temporal im Mittelfeld }\end{array}$ & $\begin{array}{l}\text { „Das Fest beginnt in fast allen Gemeinden jeweils am } 28 \text { April.“ (MM Juli, } \\
\text { 1989) }\end{array}$ \\
\hline $\begin{array}{l}\text { Temporal im Mittelfeld } \\
\text { Lokal im Mittelfeld }\end{array}$ & $\begin{array}{l}\text { „Die Abende beginnen jeweils um } 19.30 \text { im Heinrich-Pesch-Haus, } \\
\text { Frankenthaler Straße 229.“ (MM September, 1994) }\end{array}$ \\
\hline Temporal im Vorfeld & „Am Freitag beginnt die neue Eishockey-Saison.“ (MM September, 1994) \\
\hline Lokal im Vorfeld & $\begin{array}{l}\text { „Osterurlauber müssen bereits an diesem Wochenende mit Staus auf den } \\
\text { Autobahnen in der Bundesrepublik und in Österreich rechnen: In neun } \\
\text { Bundesländern sowie in Österreich, Schweden und Dänemark beginnen die }\end{array}$ \\
\hline
\end{tabular}

\footnotetext{
${ }^{75}$ Unter ,andere Satzglieder“ fasse ich das Muster ,jemand beginnt mit etwas ‘ zusammen.

${ }^{76}$ Zur Feststellung bestimmter Regelmäßigkeiten sollte man die Ergebnisse bei den Verben stattfinden und beginnen vergleichen.
} 


\begin{tabular}{|c|c|}
\hline & Schulferien."(MM März, 1989) \\
\hline $\begin{array}{l}\text { Temporal im Vorfeld } \\
\text { Temporal im Mittelfeld }\end{array}$ & „An beiden Tagen beginnt der Spielbetrieb um 10 Uhr.“(MM Juni, 1989) \\
\hline $\begin{array}{l}\text { Temporal im Vorfeld } \\
\text { Lokal im Mittelfeld }\end{array}$ & „Im November beginnt im Garten der große Kehraus.“(MM Oktober, 1994) \\
\hline $\begin{array}{l}\text { Lokal im Vorfeld } \\
\text { Temporal im Mittelfeld }\end{array}$ & $\begin{array}{l}\text { „Im Mannheimer Puppentheater am Kurpfalzkreisel beginnt am Wochenende } \\
\text { die neue Spielzeit.“ (MM Oktober, 1994) }\end{array}$ \\
\hline $\begin{array}{l}\text { Lokal im Vorfeld } \\
\text { Temporal im Mittelfeld } \\
\text { Lokal im Mittelfeld }\end{array}$ & $\begin{array}{l}\text { „Im Herzogenriedpark beginnt die Veranstaltung um } 17 \text { Uhr auf der Bühne der } \\
\text { Konzertmuschel mit dem St.-Martinspiel.“(MM November, 1989) }\end{array}$ \\
\hline $\begin{array}{l}\text { Temporal im Vorfeld } \\
\text { Temporal im Mittelfeld } \\
\text { Lokal im Mittelfeld }\end{array}$ & $\begin{array}{l}\text { „Am Sonntag, 16. Oktober, beginnt um } 13 \text { Uhr am Rathaus in Seckenheim der } \\
\text { Kulturspaziergang mit Lothar Mark.“ (MM September, 1994) }\end{array}$ \\
\hline
\end{tabular}

- Satzkonstruktion: ,Ich beginne mit etwas/Ich beginne etwas ${ }^{77}$ Transitive und präpositionale Valenzrealisierung

\begin{tabular}{||l||l||}
\hline \hline Temporal im Mittelfeld & $\begin{array}{l}\text {,Der ,Mannheimer Morgen ' beginnt heute eine Umfrage zum Thema ,Pflanzen- } \\
\text { und Tierschutz ohne Gift'.“(MM Juni, 1989) }\end{array}$ \\
\hline \multirow{3}{*}{ Temporal im Vorfeld } & $\begin{array}{l}\text {,Am Mittwoch, 8. Januar um } 15 \text { Uhr beginnt die Melanchthonakademie ihr } \\
\text { Winterprogramm mit einem Beitrag über ,Martin Butzer, ein Pfälzer } \\
\text { Reformator." (MM Januar, 1991) }\end{array}$ \\
\hline $\begin{array}{l}\text { Lokal im Vorfeld } \\
\text { April, 1989) }\end{array}$ \\
\hline
\end{tabular}

Im Weiteren befasse ich mich mit den unterschiedlichen Valenzrealisierungen sowie mit ihren entsprechenden Bedeutungsunterschieden.

1. ,etwas beginnt ${ }^{e}$,etwas passiert ${ }^{e}$, ,etwas existiert ${ }^{e}$

\begin{tabular}{|c||c|c|c|}
\hline \multicolumn{4}{|c|}{ Subjekt } \\
\hline \hline Relationale Bedeutung & Kategorielle Bedeutung & bestimmt & bekannt \\
\hline Nicht-Agens-Rolle & {$[\mathrm{akt}] /[\mathrm{stat}]$} & $+/-$ & $+/-$ \\
\hline
\end{tabular}

2. ,Ich beginne mit etwas ${ }^{e}$

\begin{tabular}{|c||c|c|c||}
\hline \multicolumn{3}{|c||}{ Subjekt } \\
\hline \hline Relationale Bedeutung & Kategorielle Bedeutung & bestimmt & bekannt \\
\hline Agens-Rolle & {$[\mathrm{hum}] /[\mathrm{inst}]$} & $+/-$ & $+/-$ \\
\hline
\end{tabular}

${ }^{77}$ Darunter werden Beispiele wie: ich beginne etwas, ich beginne mit etwas (= Ich beginne etwas zu tun) und etwas beginnt mit etwas (= etwas ist der Anfang von etwas) zusammengefasst. 
Im zweiten Fall ergeben meine Befunde, dass temporale, lokale usw. Satzglieder nur in geringer Zahl vorkommen. Dagegen ist bei der intransitiven Realisierung die Anzahl dieser Satzglieder sehr hoch.

\section{Analyse der Ergebnisse}

4.a. Durch die Beispielsammlung ist bewiesen worden, dass die Erwartbarkeit und die Vorkommenshäufigkeit dieser Satzglieder bei einigen Verben (vgl. 3.) und bei bestimmten Valenzrealisierungen sehr hoch sind. Die Festlegung einer genauen Grenze dieser Vorkommenshäufigkeit ist immerhin fraglich, aber meiner Ansicht nach sollten die Analyseergebnisse dazu führen, dass die Frage nach der Subklassenspezifität dieser Satzglieder gestellt werden soll ${ }^{78}$. Die Vorkommenshäufigkeit eines Satzgliedes in einer Beispielsammlung sollte darauf schließen lassen, dass seine ,beliebige Kombinierbarkeit vielleicht doch nicht so beliebig ist.

Um die Frage $\mathrm{zu}$ beantworten, ob die kausalen, lokalen und temporalen Rollen subklassenspezifisch sind oder nicht, muss man meines Erachtens auf Gruppen von Verben eingehen, bei denen man sich ähnliche Szenen vorstellen kann (siehe z.b. anfangen und beginnen).

4.b. Zur Bestimmung dieser Satzglieder als subklassenspezifisch oder nicht, Ergänzung oder Angabe, scheinen die Eigenschaften Nicht-Agens-Rolle und Agens-Rolle entscheidend zu sein. Bei allen hier untersuchten Verben mit dem Merkmal Nicht-Agens-Rolle kommen diese Satzglieder in einer höheren Anzahl vor. In diesem Fall sollte man sie als subklassenspezifisch verstehen, die zudem in bestimmten Szenen perspektiviert sein können oder nicht. Diese Subklassenspezifik lässt sich auch durch die unterschiedlichen Valenzrealisierungen, durch die Bedeutungsvariationen in unterschiedlichen Kontexten und Situationen und durch die morphosyntaktischen Variationen begründen, d.h. in bestimmten frames sind diese Satzglieder perspektivierungsfixiert, denn ihr Weglassen hängt nicht nur mit pragmatischen, sondern auch mit Variationen wie +/- bestimmt, +/- bekannt bei den Äußerungen zusammen. Eng verbunden mit den unterschiedlichen Valenzrealisierungen ist der Reduktionstest von Zifonun u.a. (vgl. 2.1.2.). Dabei ist $\mathrm{zu}$ beachten, ob durch das Weglassen eines Satzgliedes eine andere Valenzrealisierung entsteht. In diesem Fall erfüllt das Satzglied die Funktion einer Ergänzung. Das ist der Fall bei vielen der hier untersuchten Verben bzw. Verbrealisierungen.

4.c. Neben der Erwartbarkeits- und Vorkommenshäufigkeit habe ich ein anderes Kriterium erwähnt: die Topologie (vgl. 2.3.). Dieses Kriterium kann in Verbindung mit der Agentivität des Subjekts gebracht werden:

A. Wenn eine Nicht-Agens-Rolle vorliegt, gibt es bei Verben wie sterben und geschehen eine überwiegende Besetzung des Mittelfelds. Dies spricht für die Einstufung der hier untersuchten Satzglieder als mögliche Ergänzungen. Wenn mehrere Klassen dieser Glieder vorkommen, steht das Temporalsatzglied überwiegend am Anfang.

78 Hingegen gibt es auch Ergänzungen, die bei sehr wenigen Verben vorkommen, wie z.B. Engels Expansivergänzung (Engel: Deutsche Geammatik [Anm. 1], S. 102). 
B. Wenn eine Agens-Rolle vorliegt, gibt es bei Verben wie öffnen oder beginnen eine überwiegende Besetzung des Vorfelds. Die Funktion dieser adverbialen Satzglieder für die Text-/Diskursorganisation kann als situative Rahmensetzung beschrieben werden. Sie stehen im Mittelfeld, wenn eine Nicht-Agens-Rolle vorliegt.

Deutliche Regularitäten ergeben sich durch die gleichzeitige Betrachtung der Topologie, der Valenzrealisierungen und der Agentivität bzw. Nicht-Agentivität des Subjekts.

Zwei Fragen bleiben noch offen:

a. Warum besetzen diese Satzglieder bei einem intransitiven Gebrauch (oder im Zustandspassiv wie bei öffnen) nicht in einer höheren Anzahl das Vorfeld?

Bei den untersuchten Belegen lässt sich eine vorwiegende Mittelfeldbeseztung feststellen. Es gibt bei der Sammlung sehr wenige Belege, in denen eine Nicht-Agens-Rolle (+bestimmt) vorkommt und das Vorfeld besetzt wird, z.B. bei beginnen:

„Um 13 Uhr beginnt das Skatturnier.“ (MM August, 1989); „Im Herbst beginnt die Auslieferung.“ (MM September, 1994); ,Am Samstag beginnt die Herbstmesse.“ (MM September, 1994)

Leider ist die Topologie kein unwiderlegbares Kriterium, da die Thema-Rhema-Gliederung einen großen Einfluss darauf hat. Wenn schon bestimmte Maximen für die Topologie der Angaben und Ergänzungen akzeptiert werden können ${ }^{79}$, sollen auch Aspekte wie Kontext und Situation mitberücksichtigt werden. In diesem Sinne spielen die Merkmale ,bekannt ${ }^{e}$ oder ,unbekannt ${ }^{\bullet}$ (die ich bei der Beschreibung der Verben hinzugefügt habe) eine sehr wichtige Rolle für die Besetzung des Vor- und Mittelfeldes.

b. Warum werden bei den Verben geschehen, stattfinden und anderen, bei denen eine NichtAgens-Rolle vorkommt, die temporalen, lokalen und weiteren Satzglieder ins Mittelfeld gestellt?

In der Regel sind es die rhematischen Elemente, die die neue Information bilden. Diese Tatsache erklärt nicht nur die Regelmäßigkeiten und Unterschiede bei den Belegen, bei denen dem Subjekt keine Agentivität zugesprochen werden kann, sondern erklärt auch die unterschiedliche Topologie und Häufigkeit von Elementen in den beiden Gruppen der untersuchten Verben, d.h. bei den Verben mit einer Nicht-Agens-Rolle (+/-bestimmt) und bei den Verben mit einer Agens-Rolle.

Aufgrund der oben genannten rhematischen und thematischen Gliederungsprinzipien trägt meine topologische Betrachtung nicht entscheidend zu der Unterscheidung zwischen Angabe und Ergänzung bei. Es kann nur festgestellt werden, dass bestimmte topologische Regelmäßigkeiten bei Angaben und Ergänzungen zu bemerken sind. Das kann aber nicht als ein entscheidendes Unterscheidungskriterium angewandt werden.

\section{Abschließende Bemerkungen}

In diesem Beitrag habe ich den Versuch unternommen, syntaktische und semantische Kriterien für die Unterscheidung von situativen Angaben und situativen Ergänzungen

${ }^{79}$ vgl. Dürscheid [Anm. 39], S. 99; Andreas Lötscher: Satzgliedstellung und funktionale Satzperspektive, in: Pragmatik in der Grammatik [Anm. 41], S. 143; Zifonun u.a. [Anm. 25], S. 1642. 
festzulegen. Man sieht anhand der Belegsammung, dass bei bestimmten Verben bestimmte Satzgliedklassen im höheren Grad erwartet werden und vorkommen. Meiner Ansicht nach sollte mindestens ihr beliebiges Vorkommen in Frage gestellt werden.

Das Topologiekriterium hilft nicht dabei, Angabe und Ergänzung zu unterscheiden, dagegen rechtfertigen die unterschiedlichen Valenzrealisierungen mit einer entsprechenden Bedeutungsänderung mein Verfahren. Für die Annahme, dass diese lokalen, temporalen und weiteren Satzglieder die Funktion einer Ergänzung erfüllen, spricht die Tatsache der Interdependenzbeziehung zwischen Verb und diesen Satzgliedern, denn nach dem Reduktionstest von Zifonun u.a. veranlasst das Weglassen der temporalen, kausalen oder lokalen Aktanten eine Bedeutungsänderung.

Meiner Ansicht nach leistet die vorliegende Studie eine einheitliche Beschreibung dieser Verben und stellt neue Unterscheidungsmittel für diese strittigen Fälle dar. Das hier Dargestellte kann akzeptiert oder revidiert werden, aber der empirische Teil zeigt eindeutige Ergebnisse. 